\title{
Dynamic Models of Appraisal Networks Explaining Collective Learning*
}

\author{
Wenjun Mei Noah E. Friedkin Kyle Lewis $\quad$ Francesco Bullo ${ }^{\dagger}$
}

June 12, 2018

\begin{abstract}
This paper proposes models of learning process in teams of individuals who collectively execute a sequence of tasks and whose actions are determined by individual skill levels and networks of interpersonal appraisals and influence. The closely-related proposed models have increasing complexity, starting with a centralized manager-based assignment and learning model, and finishing with a social model of interpersonal appraisal, assignments, learning, and influences. We show how rational optimal behavior arises along the task sequence for each model, and discuss conditions of suboptimality. Our models are grounded in replicator dynamics from evolutionary games, influence networks from mathematical sociology, and transactive memory systems from organization science.
\end{abstract}

Index terms: collective learning, transactive memory systems, appraisal networks, influence networks, evolutionary games, replicator dynamics, multi-agent systems

\section{Introduction}

\subsection{Motivation and problem description}

Researchers in sociology, psychology, and organization science have long studied the inner functioning and performance of teams with multiple individuals engaged in tasks. Extensive qualitative studies, conceptual models and empirical studies in the laboratory and field reveal some statistical features and various phenom-

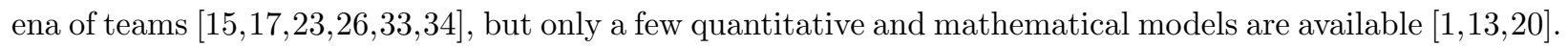
In this paper we build mathematical models for the dynamics of team structure and performance. Our work is based on the core idea that all the exhibited phenomena and features of teams must result from some essential elements, that is, individual (member) attributes and the team's inner structure. We aim to mathematically characterize these essential elements of teams, investigate how they are related to team performance and, most importantly, how they evolve with time.

\footnotetext{
*This material is based upon work supported by, or in part by, the U. S. Army Research Laboratory and the U. S. Army Research Office under grant numbers W911NF-15-1-0577 and W911NF-16-1-0005. The content of the information does not necessarily reflect the position or the policy of the Government, and no official endorsement should be inferred. A preliminary version 24] has been accepted to the 53rd IEEE Conference on Decision and Control. Compared with 24], this paper proposes more generalized models, discusses numerous model variations, and provides all the mathematical proofs absent in 24].

†Wenjun Mei and Francesco Bullo are with the Department of Mechanical Engineering and with Center for Control, Dynamical Systems, and Computation, University of California, Santa Barbara, Santa Barbara, CA 93106, USA, meiwenjunbd@gmail.com, bullo@engineering.ucsb.edu Noah E. Friedkin is with the Department of Sociology and with Center for Control, Dynamical Systems, and Computation, University of California, Santa Barbara, Santa Barbara, CA 93106, USA, friedkin@soc.ucsb.edu Kyle Lewis is with the Technology Management Program, University of California, Santa Barbara, Santa Barbara, CA 93106, USA, klewis@tmp.ucsb.edu
} 
We consider a team of individuals with unknown skill levels who complete a sequence of tasks. The team's basic inner structure is characterized by the appraisal network. The appraisal network determines how each task is assigned, and is updated via performance feedback and its co-evolution with the team's influence network. We aim to build multi-agent dynamical models in which (i) the team as an entirety eventually achieves the optimal assignment of tasks to members; (ii) each individual's true relative skill level is asymptotically learned by the team members, which is referred to as collective learning. We then investigate the model variations for what impairs collective learning.

\subsection{Literature review}

Our work is deeply connected with a conceptual model of team learning and performance, a transactive memory system (TMS). A TMS is characterized by individuals' skills and knowledge, combined with members' collective understanding of which members possess what knowledge 31. When members share an understanding of who knows what on the team, tasks can be assigned to members most likely to possess the appropriate skills. As members observe the task performances of other members, their understanding of "who knows what" tends to become more accurate and more similar, leading to greater coordination and integration of members' knowledge. Empirical research across a range of team types and settings demonstrates a strong positive relationship between the development of a team TMS and team performance 16 17, 35.

In our models, collective learning arises as the result of the co-evolution of interpersonal appraisals and influence networks. Related previous work includes social comparison theory [5], averaging-based social learning [9], opinion dynamics on influence networks [4, $8,18,25]$, reflected appraisal mechanisms [2,7|11], dynamic balance theory [22, 30, and the combined evolution of interpersonal appraisals and influence networks [10].

In the modeling and analysis of the evolution of appraisal and influence networks, we also build an insightful connection between our model and the well-known replicator dynamics studied in evolutionary game theory; see the textbook [28, some control applications [6,21], and the recent contributions [3, 19].

\subsection{Contribution}

Firstly, based on a few natural assumptions, we propose three novel models with increasing complexity for the dynamics of teams: the manager dynamics, the assign/appraise dynamics, and the assign/appraise/influence dynamics. Our work integrates three well-established types of dynamics: the replicator dynamics, the dynamics of appraisal networks, and the opinion dynamics on influence networks. To the best of our knowledge, this is the first time that such an integration has been proposed. Our models provide an insightful perspective on the connection between team performance and the interpersonal appraisal networks. In our models, the performances of a team of individuals, with fixed skill levels, are determined by how a task is assigned. For the baseline manager dynamics, task assignment is adjusted by an outside authority, according to the replicator dynamics, with individuals' performances as the feedback signals. The assign/appraise dynamics elaborates the baseline model by assuming that, instead of an outside authority, the team members' interpersonal appraisals as the basic inner structure determine the task assignments. In the assign/appraise/influence dynamics model, we further elaborate the model by considering the co-evolution of appraisal and influence networks.

Secondly, theoretical analysis is presented on the dynamical properties of the models we propose. We prove that, for the assign/appraise and the assign/appraise/influence dynamics, task assignments determined by the interpersonal appraisals satisfy the replicator dynamics in a generalized form. Moreover, results on the models' asymptotic behavior relate collective learning with the connectivity property of the observation network, which defines the heterogeneous feedback signals each individual observes. We find that, for the 
assign/appraise dynamics with the initial appraisal network that is strongly connected and ha a self loop for each node, the team achieves rational task assignment, if the observation network is strongly connected. For the assign/appraise/influence dynamics with strongly connected and aperiodic initial appraisal network, the team achieves collective learning, if the observation network has a globally reachable node. Our theoretical results on the asymptotic behavior can be interpreted as the exploration of the most relaxed condition for asymptotic optimal task assignment. In addition, the assign/appraise/influence dynamics describes an emergence process by which team members' perception of "who knows what" become more similar over time, a fundamental feature of TMS $14,27$.

Thirdly, besides the models in which the team eventually learns the individuals' true relative skill levels, we propose one variation in each of the three phases of the assign/appraise/influence dynamics: the assignment rule, the update of appraisal network based on feedback signal, and the opinion dynamics for the interpersonal appraisals. The variations reflect some sociological and psychological mechanisms known to prevent the team from learning. We investigate by simulation numerous possible causes of failure to learn.

\subsection{Organization}

The rest of this paper is organized as follows: the next subsection introduces some preliminaries on evolutionary games and replicator dynamics; Section II proposes our problem set-up and centralized manager model; Section III introduces the assign/appraise dynamics; Section IV is the assign/appraise/influence model; Section V discusses some causes of failure to learn; Section VI provides some further discussions and conclusion.

\subsection{Preliminaries}

Evolutionary games apply game theory to evolving populations adopting different strategies. Consider a game with finite pure strategies $\boldsymbol{e}_{1}, \boldsymbol{e}_{2}, \ldots, \boldsymbol{e}_{n}$ and mixed strategies $\boldsymbol{w} \in \operatorname{int}\left(\Delta_{n}\right)$. The expected payoff for mixed strategy $\boldsymbol{v}$ against mixed strategy $\boldsymbol{w}$ is defined as the payoff function $\pi(\boldsymbol{v}, \boldsymbol{w})=\sum_{i=1}^{n} v_{i} \pi_{i}(\boldsymbol{w})$, with $\pi_{i}(\boldsymbol{w})=\pi\left(\boldsymbol{e}_{i}, \boldsymbol{w}\right)$ for simplicity. A strategy $\hat{\boldsymbol{w}}$ is an evolutionary stable strategy (ESS) if any mutant strategy $\boldsymbol{v} \neq \hat{\boldsymbol{w}}$, adopted by an $\epsilon$-fraction of the population, brings less expected payoff than the majority strategy $\hat{\boldsymbol{w}}$, as long as $\epsilon$ is sufficiently small. A necessary and sufficient condition for a local ESS is stated as follows: there exists a neighborhood $U(\hat{\boldsymbol{w}})$ such that, for any $\boldsymbol{v} \in U(\hat{\boldsymbol{w}}) \backslash\{\hat{\boldsymbol{w}}\}, \pi(\hat{\boldsymbol{w}}, \boldsymbol{v})>\pi(\boldsymbol{v}, \boldsymbol{v})$.

Replicator dynamics, given by equation (1), models the evolution of sub-population distribution $\boldsymbol{w}(t) \in$ $\Delta_{n}$. Each sub-population $i$, with fraction $w_{i}(t)$ at time $t$, is using strategy $\boldsymbol{e}_{i}$ and has the growth rate proportional to its fitness, defined as the expected payoff $\pi_{i}(\boldsymbol{w}(t))$.

$$
\dot{w}_{i}=w_{i}\left(\pi_{i}(\boldsymbol{w})-\sum_{k=1}^{n} w_{k} \pi_{k}(\boldsymbol{w})\right) .
$$

The time index $t$ is omitted for simplicity. There is a simple connection between the ESS and the replicator dynamics 28]: if the payoff function $\pi(\boldsymbol{v}, \boldsymbol{w})$ is linear to $\boldsymbol{w}$, then an ESS is a globally asymptotically stable equilibrium for system (1); if $\pi(\boldsymbol{v}, \boldsymbol{w})$ is nonlinear to $\boldsymbol{w}$, then the ESS is locally asymptotically stable.

\section{Problem Set-up and Manager Dynamics}

In this section we introduce some basic formulations and a baseline centralized system on the evolution of a team. Frequently used notations are listed in Table 1 


\subsection{Model assumptions and notations}

a) Team, tasks and assignments: The basic assumption on the individuals and the tasks are given below.

Assumption 1 (Team, task type and assignment). Consider a team of $n$ individuals characterized by a fixed but unknown vector $\boldsymbol{x}=\left(x_{1}, \ldots, x_{n}\right)^{\top}$ satisfying $\boldsymbol{x} \succ \mathbb{O}_{n}$ and $\boldsymbol{x}^{\top} \mathbb{1}_{n}=1$, where each $x_{i}$ denotes the skill level of individual $i$. The tasks being completed by the team are assumed to have the following properties:

(i. The total workload of each task is characterized by a positive scalar and is fixed as 1 in this paper;

(ii. The task can be arbitrarily decomposed into $n$ sub-tasks according to the task assignment $\boldsymbol{w}=\left(w_{1}, \ldots, w_{n}\right)^{\top}$, where each $w_{i}$ is the sub-task workload assigned to individual $i$. The task assignment satisfies $\boldsymbol{w} \succ \mathbb{O}_{n}$ and $\boldsymbol{w}^{\top} \mathbb{1}_{n}=1$. The sub-tasks are executed simultaneously.

The selection of scalar values for skill levels and task assignments can be simply interpreted as the assumption that the type of tasks considered in this paper only requires some one-dimension skill. Alternatively, the skill levels can be considered more generally as the individuals' overall abilities of contributing to the completion of tasks, and the task assignments are the individuals' relative responsibilities to the team.

b) Individual performance: With fixed skill levels $\boldsymbol{x}$, each individual $i$ 's performance is assumed to depend only on the task assignment $\boldsymbol{w}$, denoted by $p_{i}(\boldsymbol{w})$. The measure of individual performance is defined below.

Assumption 2 (Individual performance). Given fixed skill levels, each individual $i$ 's performance, with the assignment $\boldsymbol{w}$, is measured by $p_{i}(\boldsymbol{w})=f\left(x_{i} / w_{i}\right)$, where $f:[0,+\infty) \rightarrow[0,+\infty)$ is a concave, continuously differentiable and monotonically increasing function.

The function $f$ is assumed to be concave in that, it is widely adopted that the relation between the performance $f$ and individual ability $x$ obeys the power law, i.e., $f(x) \sim x^{\gamma}$, with $0<\gamma<1$ [1]. Despite the specific form $f\left(x_{i} / w_{i}\right)$ as in Assumption 2 the measure of individual performance can be quite general by adopting difference measures of $x_{i}$ and $w_{i}$.

c) Optimal assignment: It is reasonable to claim that, in a well-functioning team, individuals' relative responsibilities, characterized by the task assignment in this paper, should be proportional to their actual abilities. Define the measure of the mismatch between task assignment and individual's true skill levels as $\mathcal{H}_{1}(\boldsymbol{w})=\sum_{i=1}^{n}\left|w_{i} / x_{i}-1\right|$. For fixed $\boldsymbol{x}$, the optimal assignment $\boldsymbol{w}^{*}=\boldsymbol{x}$ minimizes $\mathcal{H}_{1}(\boldsymbol{w})$.

\subsection{Centralized manager dynamics}

In this subsection we introduce a continuous-time centralized model on the evolution of task assignment, referred to as the manager dynamics. The diagram illustration is given by Figure 1(a). Suppose that, at each time $t$, a team of $n$ individuals is completing a task based on the assignment $\boldsymbol{w}(t)$, which is determined and adjusted along $t$ by an outside manager. The manager observes the individuals' real-time performance $\boldsymbol{p}(\boldsymbol{w}(t))$ and adjust the task assignment $\boldsymbol{w}(t)$ according to the dynamics:

$$
\dot{w}_{i}=w_{i}\left(p_{i}(\boldsymbol{w})-\sum_{k=1}^{n} w_{k} p_{k}(\boldsymbol{w})\right),
$$

for any $i \in\{1, \ldots, n\}$. The following theorem states the asymptotic behavior of the manager dynamics.

Theorem 1 (Manager dynamics). Consider the manager dynamics 22 for the task assignment as in Assumption 1 with performance as in Assumption 2 . Then 
Table 1: Notations frequently used in this paper

\begin{tabular}{rl}
\hline$\succ(\prec$ resp. $)$ & entry-wise greater than (less than resp.). \\
$\succeq(\preceq$ resp. $)$ & entry-wise no less than (no greater than resp.). \\
$\mathbb{1}_{n}\left(\mathbb{O}_{n}\right.$ resp. $)$ & $n$-dimension column vector with all entries equal to $1(0$ resp. $)$ \\
$\boldsymbol{x}$ & vector of individual skill levels, with $\boldsymbol{x}=\left(x_{1}, x_{2}, \ldots, x_{n}\right)^{\top} \succ \mathbb{O}_{n}$ and $\boldsymbol{x}^{\top} \mathbb{1}_{n}=1$. \\
$\boldsymbol{w}$ & task assignment. $\boldsymbol{w} \succ \mathbb{O}_{n}$ and $\boldsymbol{w}^{\top} \mathbb{1}_{n}=1$ \\
$f$ & a concave, continuously differentiable and increasing function $f:[0,+\infty) \rightarrow[0,+\infty)$ \\
$\boldsymbol{p}(\boldsymbol{w})$ & vector of individual performances. $\boldsymbol{p}(\boldsymbol{w})=\left(p_{1}(\boldsymbol{w}), \ldots, p_{n}(\boldsymbol{w})\right)^{\top}$, where $p_{i}(\boldsymbol{w})=$ \\
& $f\left(w_{i} / x_{i}\right)$ is the performance of individual $i$. \\
$A$ & appraisal matrix. $A=\left(a_{i j}\right)_{n \times n}$, where $a_{i j}$ is individual $i$ 's appraisal of $j$ 's skill level. \\
$W$ & influence matrix. $W=\left(w_{i j}\right)_{n \times n}$, where $w_{i j}$ is the weight individual $i$ assigns to $j$ 's \\
& opinion. \\
$\Delta_{n}$ & $n$-dimension simplex $\left\{\boldsymbol{y} \in \mathbb{R}_{\geq 0}^{n} \mid \boldsymbol{y}^{\top} \mathbb{1}_{n}=1\right\}$ \\
$\operatorname{int}\left(\Delta_{n}\right)$ & the interior of $\Delta_{n}$. \\
$\boldsymbol{v}_{\text {left }}(A)$ & the left dominant eigenvector of the non-negative and irreducible matrix $A$, i.e., the \\
& normalized entry-wise positive left eigenvector associated with the eigenvalue equal \\
$G(B) \quad$ to $A$ 's spectral radius. & the directed and weighted graph associated with the adjacency matrix $B \in \mathbb{R}^{n \times n}$. \\
&
\end{tabular}

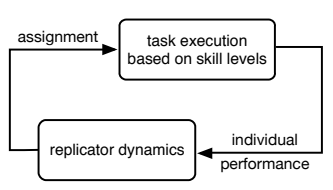

(a) manager

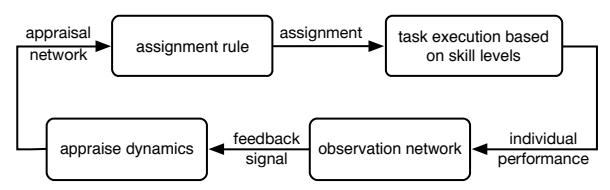

(b) assign/appraise

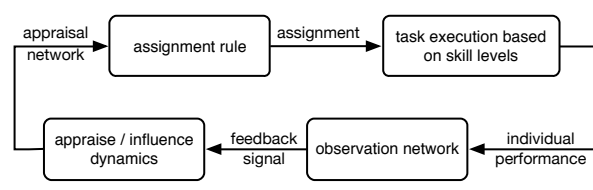

(c) assign/appraise/influence

Figure 1: Diagram illustrations of manager dynamics, assign/appraise dynamics, and assign/appraise/influence dynamics.

(i. the set $\operatorname{int}\left(\Delta_{n}\right)$ is invariant;

(ii. the optimal assignment $\boldsymbol{w}^{*}=\boldsymbol{x}$ is the ESS for the evolutionary game defined by the payoff function $\pi(\boldsymbol{v}, \boldsymbol{w})=\sum_{i=1}^{n} v_{i} f\left(x_{i} / w_{i}\right)$, and is thus a locally asymptotically stable equilibrium for equation (2) as a replicator dynamics;

(iii. for any $\boldsymbol{w}(0) \in \operatorname{int}\left(\Delta_{n}\right)$, the manager's assignment $\boldsymbol{w}(t)$ converges to $\boldsymbol{w}^{*}=\boldsymbol{x}$, as $t \rightarrow \infty$.

The proof is given in Appendix .1. Equation (2) takes the same form as the classic replicator dynamics [28], with the nonlinear fitness function $\pi_{i}(\boldsymbol{w})=f\left(x_{i} / w_{i}\right)$. The same Lyapunov function is used in the proof for the asymptotic stability. Distinct from the classic result that the ESS, with nonlinear payoff function, can only lead to local asymptotic stability for the replicator dynamics, our model is a special case in which the ESS associated with a nonlinear payoff function is also a globally asymptotically stable equilibrium of the replicator dynamics. 


\section{The Assign/Appraise Dynamics of the Appraisal Networks}

Despite the desired property on the convergence of the task assignment to optimality, the manager dynamics does not capture one of the most essential aspects of team dynamics: the evolution of the team's inner structures. In this section, we introduce a multi-agent system, in which task assignments are determined by the team members' interpersonal appraisals, rather than any outside authority, and the appraisal network is updated in a decentralized manner, driven by the heterogeneous feedback signals observed by each team member.

\subsection{Model description and problem statement}

Appraisal network: Denote by $a_{i j}$ the individual $i$ 's evaluation of $j$ 's skill levels and refer to $A=\left(a_{i j}\right)_{n \times n}$ as the appraisal matrix. Since the evaluations are in the relative sense, we assume $A \succeq \mathbb{O}_{n \times n}$ and $A \mathbb{1}_{n}=\mathbb{1}_{n}$. The directed and weighted graph $G(A)$, referred to as the appraisal network, reflects the team's collective knowledge on the distribution of its members' abilities.

Assign/appraise dynamics: We propose a multi-agent model on the evolution of the appraisal network. The model is referred to as the assign/appraise dynamics and illustrated by the diagram in Figure 1(b). We model three phases: the task assignment, the feedback signal and the update of the appraisal network, specified by the following three assumptions respectively.

Assumption 3 (Assignment rule). At any time $t \geq 0$, the task is assigned according to the left dominant eigenvector of the appraisal matrix, i.e., $\boldsymbol{w}(t)=\boldsymbol{v}_{\text {left }}(A(t))$.

Justification of Assumption 3 is given in Appendix $[2$. For now we assume $A(t)$ is row-stochastic and irreducible for all $t \geq 0$, so that $\boldsymbol{v}_{\text {left }}(A(t))$ is always well-defined. This will be proved later in this section.

Assumption 4 (Feedback signal). After executing the task assignment $\boldsymbol{w}$, each individual $i$ observes, with no noise, the difference between her own performance and the quality of some part of the whole task, given by $\sum_{k} m_{i k} p_{k}(\boldsymbol{w})$, in which $m_{i k}$ denotes the fraction of workload individual $k$ contributes to this part of task. The matrix $M=\left(m_{i j}\right)_{n \times n}$ defines a directed and weighted graph $G(M)$, referred to as the observation network, and satisfies $M \succeq \mathbb{O}_{n \times n}$ and $M \mathbb{1}_{n}=\mathbb{1}_{n}$ by construction.

The topology of the observation network defines the individuals' feedback signal structure and influences the asymptotic behavior of assign/appraise dynamics. Notice that, the feedback signal for each individual $i$ is the difference $p_{i}(\boldsymbol{w}(t))-\sum_{k} m_{i k} p_{k}(\boldsymbol{w}(t))$, while the matrix $M$ is not necessarily known to the individuals.

Assumption 5 (Update of interpersonal appraisals). With the performance feedback signal defined as in Assumption 4, each individual $i$ increases her self appraisal and decreases the appraisals of all the other individuals, if $p_{i}(\boldsymbol{w})>\sum_{k} m_{i k} p_{k}(\boldsymbol{w})$, and vice versa. In addition, the appraisal matrix $A(t)$ remains rowstochastic.

The following dynamical system for the appraisal matrix, referred to as the appraise dynamics, is arguably the simplest model satisfying Assumptions 4 and 5 .

$$
\left\{\begin{array}{l}
\dot{a}_{i i}=a_{i i}\left(1-a_{i i}\right)\left(p_{i}(\boldsymbol{w})-\sum_{k=1}^{n} m_{i k} p_{k}(\boldsymbol{w})\right), \\
\dot{a}_{i j}=-a_{i i} a_{i j}\left(p_{i}(\boldsymbol{w})-\sum_{k=1}^{n} m_{i k} p_{k}(\boldsymbol{w})\right) .
\end{array}\right.
$$


The matrix form of the appraise dynamics, together with the assignment rule as in Assumption 3 is given by

$$
\left\{\begin{array}{l}
\dot{A}=\operatorname{diag}(\boldsymbol{p}(\boldsymbol{w})-M \boldsymbol{p}(\boldsymbol{w})) A_{\mathrm{d}}\left(I_{n}-A\right), \\
\boldsymbol{w}=\boldsymbol{v}_{\text {left }}(A)
\end{array}\right.
$$

and collectively referred to as the assign/appraise dynamics. Here $A_{\mathrm{d}}=\operatorname{diag}\left(a_{11}, \ldots, a_{n n}\right)$.

Problem statement: In Section III.B, we investigate the asymptotic behavior of dynamics (4), including:

(i. convergence to the optimal assignment, which means that the team as an entirety eventually learns all its members' relative skill levels, i.e., $\lim _{t \rightarrow+\infty} \boldsymbol{w}(t)=\boldsymbol{x}$;

(ii. appraisal consensus, which means that the individuals asymptotically reach consensus on the appraisals of all the team members, i.e., $a_{i j}(t)-a_{k j}(t) \rightarrow 0$ as $t \rightarrow+\infty$, for any $i, j, k$.

Collective learning is the combination of the convergence to optimal assignment and appraisal consensus.

\subsection{Dynamical behavior of the assign/appraise dynamics}

We start by establishing that the appraisal matrix $A(t)$, as the solution to equation (4), is extensible to all $t \in[0,+\infty)$ and the assignment $\boldsymbol{w}(t)$ is well-defined, in that $A(t)$ remains row-stochastic and irreducible. Moreover, some finite-time properties are investigated.

Theorem 2 (Finite-time properties of assign/appraise dynamics). Consider the assign/appraise dynamics (4), based on Assumptions 35. describing a task assignment as in Assumption 1, with performance as in Assumption 2. For any observation network $G(M)$, and any initial appraisal matrix $A(0)$ that is row-stochastic, irreducible and has strictly positive diagonal,

(i. The appraisal matrix $A(t)$, as the solution to (4), is extensible to all $t \in[0,+\infty)$. Moreover, $A(t)$ remains row-stochastic, irreducible and has strictly positive diagonal for all $t \geq 0$;

(ii. there exists a row-stochastic irreducible matrix $C \in \mathbb{R}^{n \times n}$ with zero diagonal such that

$$
A(t)=\operatorname{diag}(\boldsymbol{a}(t))+\left(I_{n}-\operatorname{diag}(\boldsymbol{a}(t))\right) C,
$$

for all $t \geq 0$, where $\boldsymbol{a}(t)=\left(a_{1}(t), \ldots, a_{n}(t)\right)^{\top}$ and $a_{i}(t)=a_{i i}(t)$, for $i \in\{1, \ldots, n\}$;

(iii. Define the reduced assign/appraise dynamics as

$$
\left\{\begin{array}{l}
\dot{a}_{i}=a_{i}\left(1-a_{i}\right)\left(p_{i}(\boldsymbol{w})-\sum_{k=1}^{n} m_{i k} p_{k}(\boldsymbol{w})\right), \\
w_{i}=\frac{c_{i}}{\left(1-a_{i}\right)} / \sum_{k=1}^{n} \frac{c_{k}}{\left(1-a_{k}\right)}
\end{array}\right.
$$

where $\boldsymbol{c}=\left(c_{1}, \ldots, c_{n}\right)^{\top}=\boldsymbol{v}_{\text {left }}(C)$. This dynamics is equivalent to system (4) in the following sense: The matrix $A(t)$ 's each diagonal entry $a_{i i}(t)$ satisfies the dynamics (6) for $a_{i}(t)$, and, for any $t \geq 0$, $a_{i i}(t)=a_{i}(t)$ for any $i$, and $a_{i j}(t)=a_{i j}(0)\left(1-a_{i}(t)\right) /\left(1-a_{i}(0)\right)$ for any $i \neq j$;

(iv. The set $\Omega=\left\{\boldsymbol{a} \in[0,1]^{n} \mid 0 \leq a_{i} \leq 1-\zeta_{i}(\boldsymbol{a}(0))\right\}$, where $\zeta_{i}(\boldsymbol{a}(0))=\frac{c_{i}}{x_{i}} \min _{k} \frac{x_{k}}{c_{k}}\left(1-a_{k}(0)\right)$, is a compact positively invariant set for the reduced assign/appraise dynamics (6); 


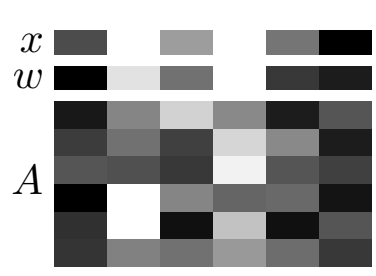

(a) $t=0$

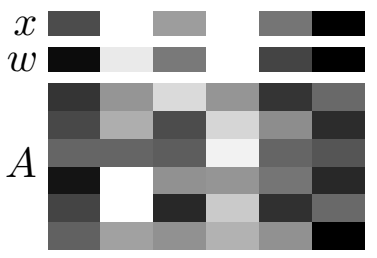

(b) $t=2$

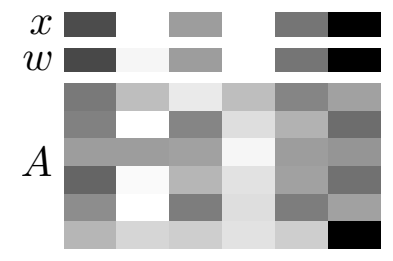

(c) $t=10$

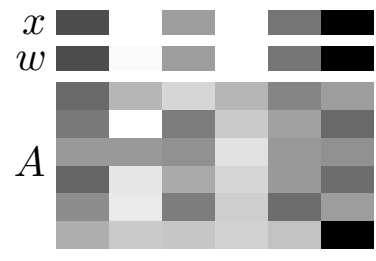

(d) $t=30$

Figure 2: Visualization of the evolution of $A(t)$ and $\boldsymbol{w}(t)$ obeying the assign/appraise dynamics with $n=6$. The observation network is strongly connected. In these visualized matrices and vectors, the darker the entry, the higher value it has.

(v. the assignment $\boldsymbol{w}(t)$ satisfies the generalized replicator dynamics with time-varying fitness function $a_{i}(t)\left(p_{i}(\boldsymbol{w}(t))-\sum_{l} m_{i l} p_{l}(\boldsymbol{w}(t))\right)$ for each $i$ :

$$
\dot{w}_{i}=w_{i}\left(a_{i}\left(p_{i}(\boldsymbol{w})-\sum_{l=1}^{n} m_{i l} p_{l}(\boldsymbol{w})\right)-\sum_{k=1}^{n} w_{k} a_{k}\left(p_{k}(\boldsymbol{w})-\sum_{l=1}^{n} m_{k l} p_{l}(\boldsymbol{w})\right)\right) .
$$

The proof for Theorem 2 is presented in Appendix .3 . With the extensibility of $A(t)$ and the finite-time properties, we now present the main theorem of this section.

Theorem 3 (Asymptotic behavior of assign/appraise dynamics). Consider the assign/appraise dynamics (4), based on Assumptions 3.5. with the task assignment as in Assumption 1 and the performance as in Assumption 2. Assume the observation network $G(M)$ is strongly connected. For any initial appraisal matrix A(0) that is row-stochastic, irreducible and has positive diagonal,

(i. the solution $A(t)$ converges, i.e., there exists $A^{*} \in \mathbb{R}^{n \times n}$ such that $\lim _{t \rightarrow \infty} A(t)=A^{*}$;

(ii. the limit appraisal matrix $A^{*}$ is row-stochastic and irreducible. Moreover, the task assignment satisfies $\lim _{t \rightarrow \infty} \boldsymbol{w}(t)=\boldsymbol{v}_{\text {left }}\left(A^{*}\right)=\boldsymbol{x}$.

The proof is presented in Appendix .4 . Theorem 3 indicates that, the teams obeying the assign/appraise dynamics asymptotically achieves the optimal task assignment, but do not necessarily reach appraisal consensus. Figure 2 gives a visualized illustration of the asymptotic behavior of the assign/appraise dynamics.

Remark 4. From the proof for Theorem 3 we know that, the teams obeying the following dynamics

$$
\left\{\begin{array}{l}
\dot{a}_{i i}=\gamma_{i}(t) a_{i i}\left(1-a_{i i}\right)\left(p_{i}(\boldsymbol{w})-\sum_{k} m_{i k} p_{k}(\boldsymbol{w})\right), \\
\dot{a}_{i j}=-\gamma_{i}(t) a_{i i} a_{i j}\left(p_{i}(\boldsymbol{w})-\sum_{k} m_{i k} p_{k}(\boldsymbol{w})\right),
\end{array}\right.
$$

also asymptotically achieve the optimal assignment, if each $\gamma_{i}(t)$ remains strictly bounded from 0 . This result indicates that our model can be generalized to the case of heterogeneous sensitivities to performance feedback.

\section{The Assign/appraise/influence Dynamics of the Appraisal Net- works}

In this section we further elaborate the assign/appraise dynamics by assuming that the appraisal network is updated via not only the performance feedback, but also its co-evolution with the team members' interper- 
sonal influences. In other words, we include an opinion dynamics process among the individuals who discuss and possibly reach consensus on the values of interpersonal appraisals.

\subsection{Model description}

The new model, named the assign/appraise/influence dynamics, is defined by three components: the assignment rule as in Assumption 3 , the appraise dynamics based on Assumptions 4 and 5 and the influence dynamics, which is the opinion exchanges among individuals on interpersonal appraisals. Denote by $w_{i j}$ the weight individual $i$ assigns to $j$ (including self weight $w_{i i}$ ) in the opinion exchange. The matrix $W=\left(w_{i j}\right)_{n \times n}$ defines a directed and weighted graph, referred to as the influence network, is row-stochastic and possibly time-varying.

The diagram illustration of assign/appraise/influence dynamics is presented in Figure 1(c), and the general form is given as follows:

$$
\left\{\begin{array}{l}
\dot{A}=\frac{1}{\tau_{\text {ave }}} F_{\text {ave }}(A, W)+\frac{1}{\tau_{\text {app }}} F_{\text {app }}(A, \boldsymbol{w}), \\
\boldsymbol{w}=\boldsymbol{v}_{\text {left }}(A) .
\end{array}\right.
$$

The time index $t$ is omitted for simplicity. The term $F_{\text {app }}(A, \boldsymbol{w})$ corresponds to the appraise dynamics given by the right-hand side of the first equation in (4), while the term $F_{\text {ave }}(A, W)$ corresponds to the influence dynamics specified by the assumption below. Parameters $\tau_{\text {ave }}$ and $\tau_{\text {app }}$ are positive, and relate to the time scales of influence dynamics and appraise dynamics respectively.

Assumption 6 (Influence dynamics). For the assign/appraise/influence dynamics, assume that, at each time $t \geq 0$, the influence network is identical to the appraisal network, i.e., $W(t)=A(t)$. Moreover, assume that the individuals obey the classic DeGroot opinion dynamics [4] for the interpersonal appraisals, i.e., $F_{\text {ave }}(W, A)=-\left(I_{n}-W\right) A$.

Based on equation (8) and Assumptions 366, the assign/appraise/influence dynamics is written as

$$
\left\{\begin{array}{l}
\dot{A}=\frac{1}{\tau_{\text {ave }}}\left(A^{2}-A\right)+\frac{1}{\tau_{\text {app }}} \operatorname{diag}(\boldsymbol{p}(\boldsymbol{w})-M \boldsymbol{p}(\boldsymbol{w})) A_{\mathrm{d}}\left(I_{n}-A\right), \\
\boldsymbol{w}=\boldsymbol{v}_{\text {left }}(A)
\end{array}\right.
$$

In the next subsection, we relate the topology of the observation network $G(M)$ to the asymptotic behavior of the assign/appraise/influence dynamics, i.e., the convergence to optimal assignment and the appraisal consensus.

\subsection{Dynamical behavior of the assign/appraise/influence dynamics}

The following lemma shows that, for the assign/appraise/influence dynamics, we only need to consider the all-to-all initial appraisal network.

Lemma 5 (entry-wise positive for initial appraisal). Consider the assign/appraise/influence dynamics (9) based on Assumptions 3, 6, with the task assignment and performance as in Assumptions 1 and 2 respectively. For any initial appraisal matrix $A(0)$ that is primitive and row-stochastic, there exists $\Delta t>0$ such that $A(t) \succ \mathbb{O}_{n \times n}$ for any $t \in(0, \Delta t]$.

The proof is given in Appendix .5. Before discussing the asymptotic behavior, we state a technical assumption. 


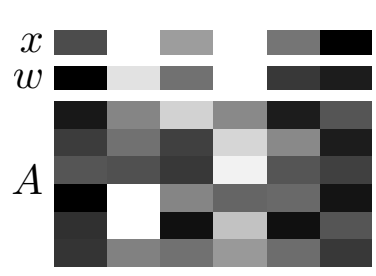

(a) $t=0$

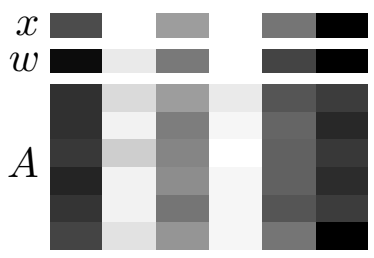

(b) $t=2$

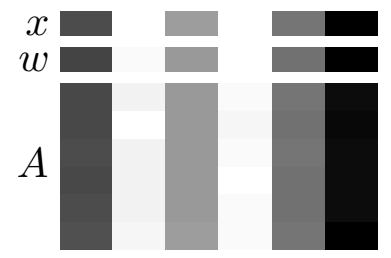

(c) $t=10$

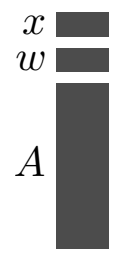

(d) $t=30$

Figure 3: Visualization of the evolution of $A(t)$ and $\boldsymbol{w}(t)$ obeying the assign/appraise/influence dynamics with $n=6$. The observation network contains a globally reachable node. In these visualized matrices and vectors, the darker the entry, the higher value it has.

Conjecture 6 (Strict lower bound of the interpersonal appraisals). Consider the assign/appraise/influence dynamics (9) based on Assumptions 3, 6, with the task assignment and performance as in Assumptions 1 and 2 respectively. For any $A(0)$ that is entry-wise positive and row-stochastic, there exists $a_{\min }>0$, depending on $A(0)$, such that $A(t) \succ a_{\min } \mathbb{1}_{n} \mathbb{1}_{n}^{\top}$ for any time $t \geq 0$, as long as $A(\tau)$ and $\boldsymbol{w}(\tau)$ are well-defined for all $\tau \in[0, t]$.

Monte Carlo validation and a sufficient condition for Conjecture 6 are presented in Appendix .6 . Now we state the main results of this section.

Theorem 7 (Assign/appraise/influence dynamical behavior). Consider the assign/appraise/influence dynamics (9) based on Assumptions 36, with the task assignment and performance as in Assumptions 1 and Assumption 2 respectively. Suppose that Conjecture 6 holds. Assume that the observation network $G(M)$ contains a globally reachable node. For any initial appraisal matrix $A(0)$ that is entry-wise positive and row-stochastic,

(i. the solution $A(t)$ exists and $\boldsymbol{w}(t)=\boldsymbol{v}_{\text {left }}(A(t))$ is well-defined for all $t \in[0,+\infty)$. Moreover, $A(t) \succ$ $\mathbb{O}_{n \times n}$ and $A(t) \mathbb{1}_{n}=\mathbb{1}_{n}$ for any $t \geq 0 ;$

(ii. the assignment $\boldsymbol{w}(t)$ obeys the generalized replicator dynamics $(7)$, and $\xi_{0} \mathbb{1}_{n} \preceq \boldsymbol{w}(t) \preceq\left(1-(n-1) \xi_{0}\right) \mathbb{1}_{n}$, where

$$
\xi_{0}=\left(1+(n-1) \frac{\max _{k} x_{k}}{\min _{l} x_{l}} \gamma_{0}\right)^{-1}, \quad \text { and } \quad \gamma_{0}=\frac{\max _{k} x_{k} / w_{k}(0)}{\min _{l} x_{l} / w_{l}(0)}
$$

(iii. as $t \rightarrow+\infty, A(t)$ converges to $\mathbb{1}_{n} \boldsymbol{x}^{\top}$ and thereby $\boldsymbol{w}(t)$ converges to $\boldsymbol{x}$.

The proof is given in Appendix .7. As Theorem 7 indicates, the team obeying the assign/appraise/ influence dynamics achieves collective learning. A visualized illustration of the dynamics is given by Figure 3.

\section{Model Variations: Causes of Failure to Learn}

The baseline assign/appraise/influence dynamics (9) consists of three phases: the assignment rule, the appraise dynamics, and the influence dynamics. In this section, we propose one variation in each of the three phases, based on some socio-psychological mechanisms that may cause a failure in team learning. We investigate the behavior of each model variation by numerical simulation.

a) Variation in the assignment rule: task assignment based on degree centrality: In Assumption 3 , the task assignment is based on the individuals' eigenvector centrality in the appraisal network. If we assume 

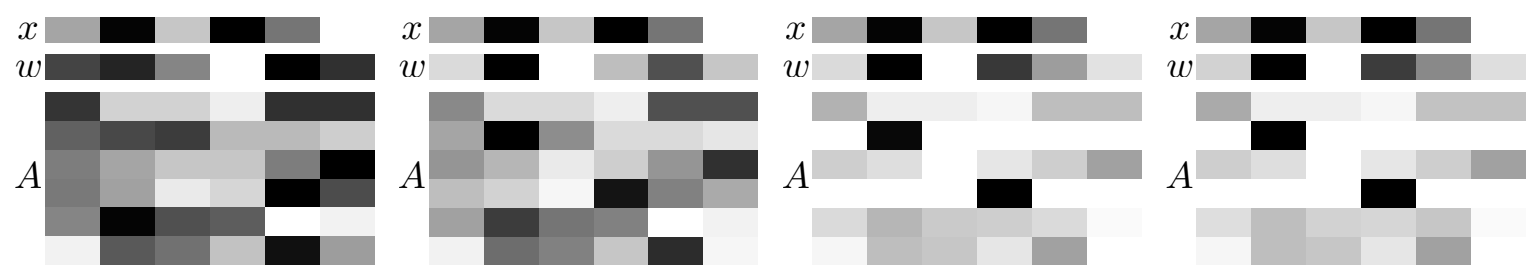

(a) no influence dynamics,

(b) no influence dynamics,

(c) no influence dynamics,

(d) no influence dynamics, $t=0$

$t=2$

$t=30$

$t=50$

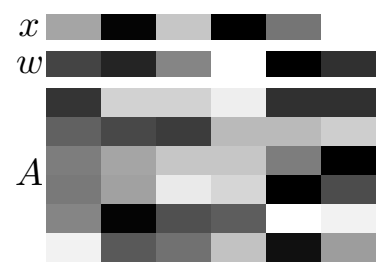

(e) with influence dynamics, (f) with influence dynamics, $t=0$

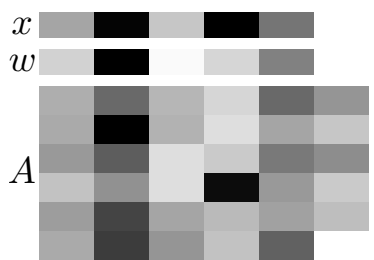

$t=2$

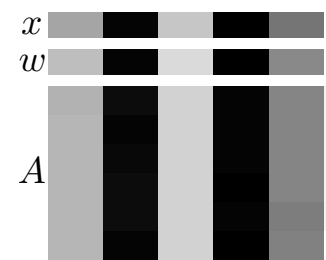

$t=30$

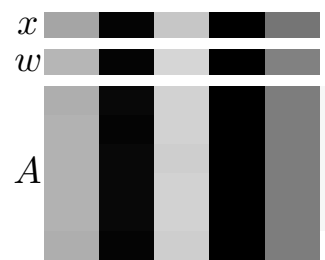

, (h) with influence dynamics, $t=50$

Figure 4: Examples of the assign/appraise (first row) and the assign/appraise/influence (second row) dynamics in which the assignment is based on the individuals' in-degree centrality. The assign/appraise dynamics does not achieve the collective learning, while the assign/appraise/influence dynamics does.

instead that the assignment is based on the individuals' normalized in-degree centrality in the appraisal network, i.e., $\boldsymbol{w}(t)=A^{\top}(t) \mathbb{1}_{n} / \mathbb{1}_{n}^{\top} A(t) \mathbb{1}_{n}$, then the numerical simulation, see Figure 4 shows the following results: the team obeying the assign/appraise dynamics does not necessarily achieve collective learning, while the team obeying the assign/appraise/influence dynamics still achieves both collective learning and appraisal consensus.

b) Variation in the appraise dynamics: partial observation of performance feedback: According to Assumption 4 , the observation network $G(M)$ determines the feedback signals received by each individual. If the observation network does not have the desired connectivity property, the individuals do not have sufficient information to achieve collective learning. Simulation results in Figure 5 shows that, if $G(M)$ is not strongly connected for the assign/appraise dynamics, or if $G(M)$ does not contain a globally reachable node for the assign/appraise/influence dynamics, the team does not necessarily achieve collective learning.

c) Variation in the influence dynamics: prejudice model: In Assumption 6, we assume that the individuals obey the DeGroot opinion dynamics. If we instead adopt the Friedkin-Johnsen opinion dynamics, given by

$$
F_{\text {ave }}(A, W)=-\Lambda\left(I_{n}-W\right) A+\left(I_{n}-\Lambda\right)(A(0)-A),
$$

where $\Lambda=\operatorname{diag}\left(\lambda_{1}, \ldots, \lambda_{n}\right)$ and each $\lambda_{i}$ characterizes individual $i$ 's attachment to her initial appraisals. Numerical simulation, see Figure 6, shows that the team does not necessarily achieve collective learning. The Friedkin-Johnsen model captures the social-psychological mechanism in which individuals show an attachment to their initial opinions. This attachment is a cause of failure of collective learning. 

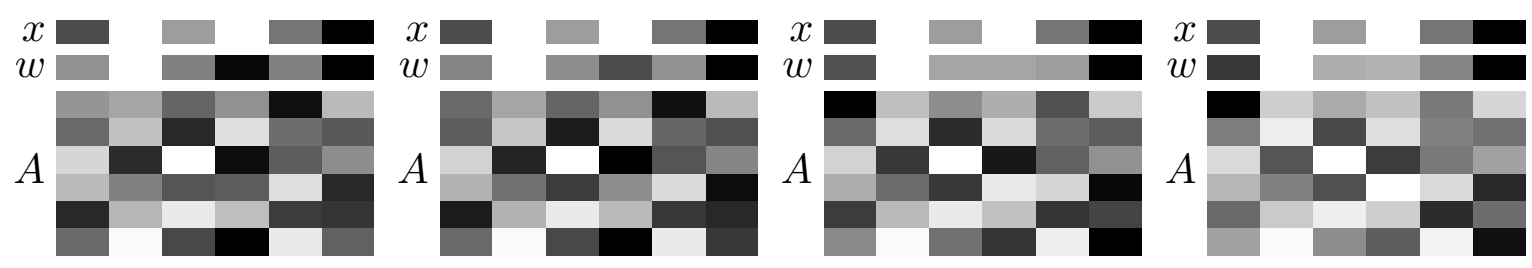

(a) no influence dynamics, (b) no influence dynamics, (c) no influence dynamics, (d) no influence dynamics,

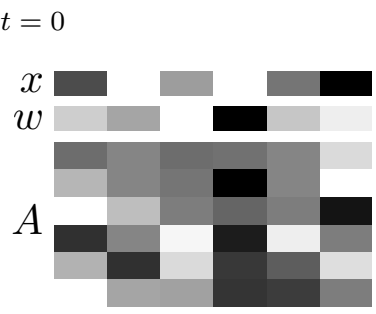
$t=1$ $t=5$ $t=10$

(e) with influence dynamics, (f) with influence dynamics, (g) with influence dynamics, (h) with influence dynamics, $t=0 \quad t=5 \quad t=50 \quad t=60$

Figure 5: Examples of failure to learn with partial observation for a six-individual team. The figures in the first row correspond to the assign/appraise dynamics, in which the observation network is not strongly connected but contains a globally reachable node. The figures in the second row correspond to the assign/appraise/influence dynamics, in which the observation network does not contain a globally reachable node. In both cases, $A(t)$ converges but $\lim _{t \rightarrow+\infty} \boldsymbol{w}(t) \neq \boldsymbol{x}$.

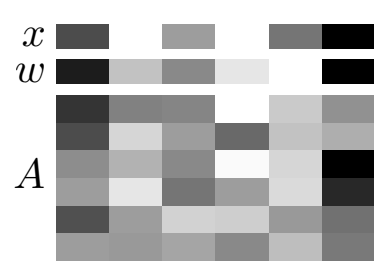

(a) $t=0$

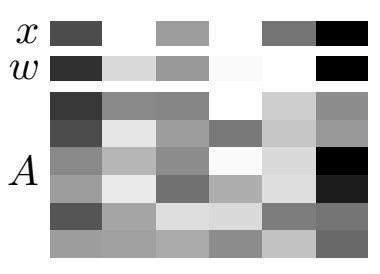

(b) $t=1$

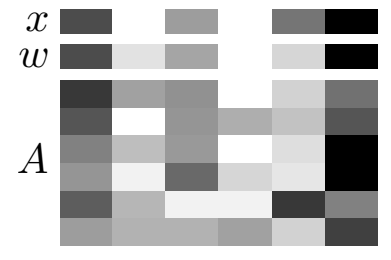

(c) $t=5$

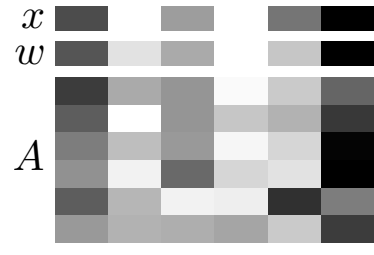

(d) $t=10$

Figure 6: Example of the evolution of $A(t)$ and $\boldsymbol{w}(t)$ in the prejudice model with $n=6$. The darker the entry, the higher value it has. The simulation result shows that $A(t)$ converges but $\boldsymbol{w}(t)=\boldsymbol{v}_{\text {left }}(A(t))$ does not necessarily converges to $\boldsymbol{x}$. 


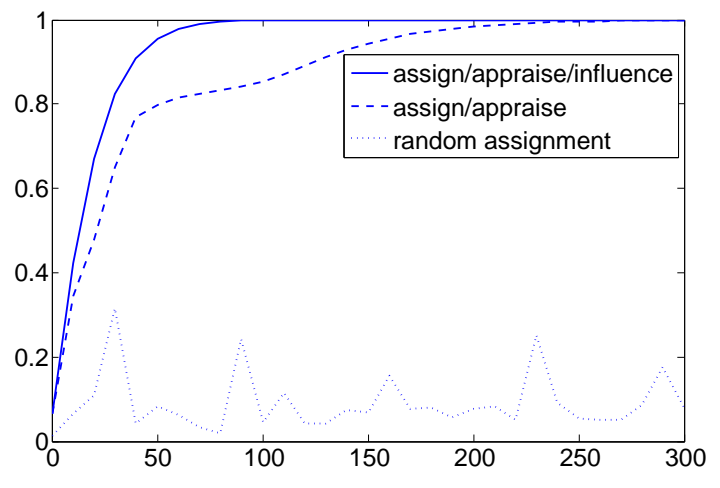

(a) $e^{-\mathcal{H}_{1}(\boldsymbol{w}, \boldsymbol{x})}$

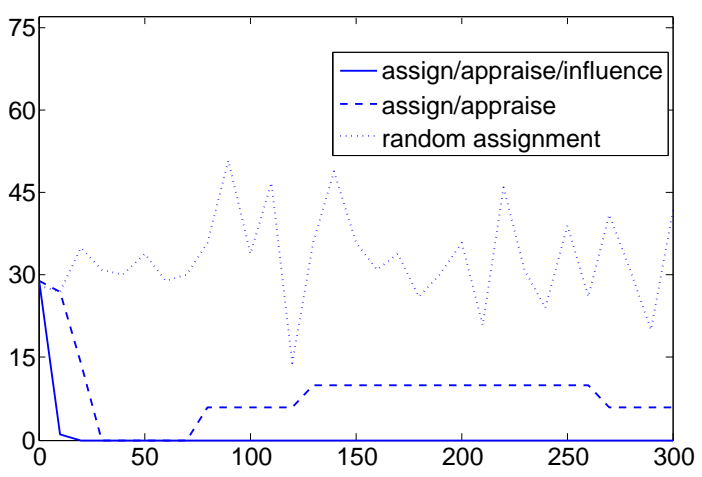

(b) Number of non-transitive triads

Figure 7: Evolution of the measure of mismatch between assignment and individual skill levels, and the number of non-transitive triads in the comparative appraisal graph. The solid curves represent the team obeying the assign/appraise/influence dynamics. The dash curves represent the team obeying the assign/appraise dynamics. The dotted curves represent the team that randomly assign sub-task workloads.

\section{Further Discussion and Conclusion}

\subsection{Connections with TMS theory}

TMS structure: As discussed in the introduction, one important aspect of TMS is the members' shared understanding about who possess what expertise. For the case of one-dimension skill, TMS structure is approximately characterized by the appraisal matrix and thus the development of TMS corresponds to the collective learning on individuals' true skill levels. Simulation results in Figure 7 compare the evolution of some features among the teams obeying the assign/appraise/influence model, the assign/appraise model, and the team that randomly assigns the sub-tasks, respectively. Figure 7(a) shows that, for both the assign/appraise/influence dynamics and the assign/appraise dynamics, function $\mathcal{H}_{1}(\boldsymbol{w}, \boldsymbol{x})$, as the measure of the mismatch between task assignment and individual skill levels, converge to 0 , which exhibits the advantage of a developing TMS.

Transitive triads: As Palazzolo 27] points out, transitive triads are indicative of a well-organized TMS. The underlying logic is that inconsistency of interpersonal appraisals lowers the efficiency of locating the expertise and allocating the incoming information. In order to reveal the evolution of triad transitivity in our models, we define an unweighted and directed graph, referred to as the comparative appraisal graph $\widetilde{G}(A)=(V, E)$, with $V=\{1, \ldots, n\}$, as follows: for any $i, j \in V,(i, j) \in E$ if $a_{i j} \geq a_{i i}$, i.e., if individual $i$ thinks $j$ has no lower skill level than $i$ herself. We adopt the standard notion of triad transitivity and use the number of non-transitive triads as the indicator of inconsistency in a team. Figure 7(b) shows that, the non-transitive triads vanish in the team obeying the assign/appraise/influence dynamics, but persist in the teams obeying the assign/appraise dynamics or just randomly assigning subtasks.

\subsection{Minimum condition for convergence to optimal assignment}

Analysis of the asymptotic behavior of assign/appraise dynamics, assign/appraise/influence dynamics and partial observation model can be interpreted as the exploration of the most relaxed condition for the convergence to optimal task assignment, concluded as follows: 
(i. Each individual only needs to know, as feedback, the difference between her own performance and the quality of some parts of the entire task, but do not need to know whom they are compared with;

(ii. The individuals can have heterogeneous but strictly positive sensitivities to the performance feedback;

(iii. The exchange of opinions on the interpersonal appraisals is not necessary;

(iv. With opinion exchange, the observation network with one globally reachable node is sufficient for the convergence to optimal assignment;

(v. Without opinion exchange, strongly connected observation network is sufficient for the convergence to optimal assignment.

\subsection{Conclusion}

This paper proposes a baseline model: the centralized manager dynamics, and two elaborative multi-agent models on team dynamics: the assign/appraise and the assign/appraise/influence dynamics. We reveal insightful connections between our models and the replicator dynamics in evolutionary game theory. For the multi-agents models, the appraisal network is modeled as a team's basic inner structure. the appraisal network generates the team's task assignments, and the mismatch between the assignment and individuals' true skill levels might indicate the level of team performance. By theoretical analysis we investigate the evolution of appraisal network, and relate its asymptotic behavior, i.e., the convergence to optimal assignment and the appraisal consensus, with the individuals' feedback signal structures. We then propose some variations of the baseline models, in which some sociological and psychological mechanisms, e.g., the assignment by in-degree centrality in the appraisal network, the prejudice in opinion dynamics, and the lack of the desired connectivity property for the observation network, cause the failure of collective learning. In addition, we show that the qualitative predictions made by our models are consistent with TMS theory in organization science.

\section{$.1 \quad$ Proof for Theorem 1}

The vector form of equation (2) is written as

$$
\dot{\boldsymbol{w}}=\operatorname{diag}(\boldsymbol{w})\left(\boldsymbol{p}(\boldsymbol{w})-\boldsymbol{w}^{\top} \boldsymbol{p}(\boldsymbol{w}) \mathbb{1}_{n}\right) .
$$

Left multiply both sides by $\mathbb{1}_{n}^{\top}$. We get $d\left(\mathbb{1}_{n}^{\top} \boldsymbol{w}\right) / d t=0$.

Since the function $f$ is continuously differentiable, the right-hand side of equation 10 is continuously differentiable and locally Lipschitz in $\operatorname{int}\left(\Delta_{n}\right)$. Define

$$
V(\boldsymbol{w})=-\sum_{i=1}^{n} x_{i} \log \frac{w_{i}}{x_{i}}
$$

We have $V(\boldsymbol{w}) \geq 0$ for any $\boldsymbol{w} \in \Delta_{n}$, due to the concavity of $\log$ function, and $V(\boldsymbol{w})=0$ if and only if $\boldsymbol{w}=\boldsymbol{x}$. Moreover, since $V(\boldsymbol{w})$ is continuously differentiable in $\boldsymbol{w}$, the level set $\left\{\boldsymbol{w} \in \operatorname{int}\left(\Delta_{n}\right) \mid V(\boldsymbol{w})=\xi\right\}$ is a compact subset of $\operatorname{int}\left(\Delta_{n}\right)$. Along the trajectory,

$$
\frac{d V(\boldsymbol{w})}{d t}=-\sum_{i \in \theta_{1}(\boldsymbol{w})}\left(x_{i}-w_{i}\right) f\left(x_{i} / w_{i}\right)-\sum_{i \in \theta_{2}(\boldsymbol{w})}\left(x_{i}-w_{i}\right) f\left(x_{i} / w_{i}\right)<0,
$$

where $\theta_{1}(\boldsymbol{w})=\left\{i \mid x_{i} \geq w_{i}\right\}$ and $\theta_{2}(\boldsymbol{w})=\left\{i \mid x_{i}<w_{i}\right\}$. This concludes the proof for the invariant set and the asymptotic stability of $\boldsymbol{w}^{*}=\boldsymbol{x}$, and one can infer, from the inequality above, that $\boldsymbol{w}^{*}=\boldsymbol{x}$ is the ESS 
for the evolutionary game with the payoff function $\pi_{i}(\boldsymbol{w})=f\left(x_{i} / w_{i}\right)$. Moreover, since $V(\boldsymbol{w}) \rightarrow+\infty$ as $\boldsymbol{w}$ tends to the boundary of $\Delta_{n}$, the region of attraction is $\operatorname{int}\left(\Delta_{n}\right)$.

\section{.2 Justifications of Assumption 3 on task assignment}

We provide some justification of Assumption.2 that, the task assignment $\boldsymbol{w}$ is given by $\boldsymbol{w}=\boldsymbol{v}_{\text {left }}(A)$. Firstly, the entries of $\boldsymbol{v}_{\text {left }}(A)$ correspond to the individuals' eigenvector centrality in the appraisal network and thus reflect how much each individual is appraised by the team. Secondly, there is a natural way in which the interpersonal interactions lead to the assignment $\boldsymbol{w}(t)=\boldsymbol{v}_{\text {left }}(A(t))$. Assume that for any incoming task, at step $k=0$, each individual $i$ evenly get $1 / n$ workload, and at each step, each individual $i$ passes $a_{i j}(t)$ of the workload she currently possesses to each individual $j \in\{1, \ldots, n\}$, until the substask distribution reaches the steady state. Denote by $q_{i}(k)$ the fraction of the workload at individual $i$, at step $k$, this workload distribution process is given by $\boldsymbol{q}(k+1)=A(t)^{\top} \boldsymbol{q}(k)$. According to Perron-Frobenius theorem, $\boldsymbol{q}(k)$ converges to $\boldsymbol{v}_{\text {left }}(A(t))$; Thirdly, our eigenvector assignment rule has the following natural property: in a team deprived of performance feedback as the information inflow, the team's task assignment does not change. In other words, along the assign/appraise/influence dynamics with $\frac{1}{\tau_{\text {app }}}=0$, vector $\boldsymbol{v}_{\text {left }}(A(t))$ remains unchanged. These arguments justify Assumption 3 recall also Section 5 (a) with a numerical evaluation of a different assignment rule.

\section{.3 Proof for Theorem 2}

Before the proof, we state a useful lemma summarized from the argument on Page 62-67 of [32].

Lemma 8 (Continuity of eigenvalue and eigenvector). Suppose $A, B \in \mathbb{R}^{n \times n}$ satisfy $\left|a_{i j}\right|<1$ and $\left|b_{i j}\right|<1$ for any $i, j \in\{1, \ldots, n\}$. For sufficiently small $\epsilon>0$,

(i. the eigenvalues $\lambda$ and $\lambda^{\prime}$ of $A$ and $(A+\epsilon B)$, respectively, can be put in one-to-one correspondence so that $\left|\lambda^{\prime}-\lambda\right|<2(n+1)^{2}\left(n^{2} \epsilon\right)^{\frac{1}{n}}$;

(ii. if $\lambda$ is a simple eigenvalue of $A$, then the corresponding eigenvalue $\lambda(\epsilon)$ of $A+\epsilon B$ satisfies $|\lambda(\epsilon)-\lambda|=$ $O(\epsilon)$;

(iii. if $\boldsymbol{v}$ is an eigenvector of $A$ associated with a simple eigenvalue $\lambda$, then the eigenvector $\boldsymbol{v}(\epsilon)$ of $A+\epsilon B$ associated with the corresponding eigenvalue $\lambda(\epsilon)$ satisfies $\left|v_{i}(\epsilon)-v_{i}\right|=O(\epsilon)$ for any $i \in\{1, \ldots, n\}$.

Proof of Theorem 2: In this proof, we extend the definition of $\boldsymbol{v}_{\text {left }}(A)$ to the normalized entry-wise positive left eigenvector, associated with the eigenvalue of $A$ with the largest magnitude, if such an eigenvector exists and is unique. According to Perron-Frobenius theorem and Lemma 8 , vector $\boldsymbol{v}_{\text {left }}(A)$, as long as welldefined, depends continuously on the entries of $A$. Therefore, for system (4), there exists a sufficiently small $\tau>0$ such that $A(t)$ and $\boldsymbol{w}(t)$ are well-defined and continuously differentiable at any $t \in[0, \tau]$, and, moreover, $p_{i}(\boldsymbol{w}(t))-\sum_{k} m_{i k} p_{k}(\boldsymbol{w}(t))$ remains finite. Therefore, for any $t \in[0, \tau]$ and $i, j \in\{1, \ldots, n\}$, $a_{i j}(t)>0$ if $a_{i j}(0)>0 ; a_{i j}(t)=0$ if $a_{i j}(0)=0$, and thus $A(t)$ is row-stochastic and primitive for any $t \in[0, \tau]$.

For any $i \in\{1, \ldots, n\}$, there exists $k \neq i$ such that $a_{i k}(0)>0$. According to equation (3),

$$
\frac{d a_{i j}(t)}{d a_{i k}(t)}=\frac{a_{i j}(t)}{a_{i k}(t)}, \forall t \in[0, \tau], \forall j \in\{1, \ldots, n\} \backslash\{i, k\},
$$

which leads to $a_{i j}(t) / a_{i k}(t)=a_{i j}(0) / a_{i k}(0)$. Let $C$ be an $n \times n$ matrix with the entries $c_{i j}$ defined as: (i) $c_{i i}=0$ for any $i \in\{1, \ldots, n\}$; (ii) $c_{i j}=a_{i j}(0) /\left(1-a_{i i}(0)\right)$ for any $j \neq i$. One can check that $C$ is 
row-stochastic and $A(t)$ is given by equation (5), for any $t \in[0, \tau]$, where $\boldsymbol{a}(t)=\left(a_{1}(t), \ldots, a_{n}(t)\right)^{\top}$ with $a_{i}(t)=a_{i i}(t)$. Since the digraph, with $C$ as the adjacency matrix, has the same topology with the digraph associated with $A(0)$, matrix $C$ is irreducible and $\boldsymbol{c}=\boldsymbol{v}_{\text {left }}(C)$ is well-defined.

Since the matrix $A(t)$ has the structure given by (5), according to Lemma 2.2 in [11], for any $t \in[0, \tau]$,

$$
w_{i}(t)=\frac{c_{i}}{1-a_{i}(t)} / \sum_{k} \frac{c_{k}}{1-a_{k}(t)} \text {. }
$$

Therefore, for any $t \in[0, \tau]$,

$$
p_{i}(\boldsymbol{w}(t))=f\left(\frac{x_{i}}{c_{i}}\left(1-a_{i}(t)\right) \sum_{k} w_{k}(t) \frac{c_{k}}{1-a_{k}(t)}\right) .
$$

According to equation (3), $\dot{a}_{j}(t) \leq 0$ for any $j \in \operatorname{argmin}_{k} \frac{x_{k}}{c_{k}}\left(1-a_{k}(t)\right)$. Therefore, $\operatorname{argmin}_{k} \frac{x_{k}}{c_{k}}\left(1-a_{k}(t)\right)$ is increasing, and similarly, $\operatorname{argmax}_{k} \frac{x_{k}}{c_{k}}\left(1-a_{k}(t)\right)$ is decreasing with $t$, which implies that, the set

$$
\Omega_{A}(A(0))=\left\{A \in \mathbb{R}^{n \times n} \mid A=\operatorname{diag}(\boldsymbol{a})+(I-\operatorname{diag}(\boldsymbol{a})) C, \quad 0 \leq a_{i} \leq 1-\frac{c_{i}}{x_{i}} \min _{k} \frac{x_{k}}{c_{k}}\left(1-a_{k k}(0)\right), \forall i\right\}
$$

is a compact positive invariant set for system (4), as long as $A(0)$ is row-stochastic, irreducible and has strictly positive diagonal. Moreover, one can check that, for any $A \in \Omega_{A}(A(0)), \boldsymbol{w}=\boldsymbol{v}_{\text {left }}(A)$ is well-defined and strictly lower (upper resp.) bounded from 0 (1 resp.). Therefore, the solution $A(t)$ is extensible to all $t \in[0,+\infty)$ and equations (5) and (6) hold for any $t \in[0,+\infty)$. Moreover, since $p_{i}(\boldsymbol{w}(t))-\sum_{k} m_{i k} p_{k}(\boldsymbol{w}(t))$ remains bounded, we have $a_{i j}>0$ if $a_{i j}(0)>0$ and $a_{i j}(t)=0$ if $a_{i j}(0)=0$. This concludes the proof for (i) - (iv).

For statement (v), differentiate both sides of the equation $\boldsymbol{w}^{\top}(t) A(t)=\boldsymbol{w}^{\top}(t)$ and substitute equation (4) into the differentiated equation. We obtain

$$
\left(\boldsymbol{w}^{\top} \operatorname{diag}(\boldsymbol{p}(\boldsymbol{w})-M \boldsymbol{p}(\boldsymbol{w})) A_{d}-\frac{d \boldsymbol{w}^{\top}}{d t}\right)\left(I_{n}-A\right)=\mathbb{O}_{n}^{\top},
$$

where time index $t$ is omitted for simplicity. Equation (7) in (v) is obtained due to $\boldsymbol{w}^{\top}(t) \mathbb{1}_{n}=1$.

\section{.4 Proof for Theorem 3}

We prove the theorem by analyzing the generalized replicator dynamics 77 for $\boldsymbol{w}(t)$, and the reduced assign/appraise dynamics (6) for $\boldsymbol{a}(t)$, given any constant, normalized and entry-wise positive vector $\boldsymbol{c}$. According to equation (6), the assignment $\boldsymbol{w}=\boldsymbol{v}_{\text {left }}(A)$ can be considered as a function of the self appraisal vector $\boldsymbol{a}$, that is, $\boldsymbol{w}(t)=\boldsymbol{w}(\boldsymbol{a}(t))$ for any $t \geq 0$. In this proof, let $\boldsymbol{\phi}(\boldsymbol{a})=\boldsymbol{p}(\boldsymbol{w}(\boldsymbol{a}))-M \boldsymbol{p}(\boldsymbol{w}(\boldsymbol{a}))$ and denote by $\mathcal{D}: \mathbb{R}^{n} \times \mathbb{R}^{n} \rightarrow \mathbb{R}_{\geq 0}$ the distance induced by the 2-norm in $\mathbb{R}^{n}$. For any $\boldsymbol{x} \in \mathbb{R}^{n}$ and subset $S$ of $\mathbb{R}^{n}$, defined $\mathcal{D}(\boldsymbol{x}, S)=\inf _{\boldsymbol{y} \in S} \mathcal{D}(\boldsymbol{x}, \boldsymbol{y})$.

First of all, for any given $\boldsymbol{a}(0) \in(0,1)^{n}$, we know that the set $\Omega$, as defined in Theorem 2 (iv), is a compact positively invariant set for dynamics (6), and $\boldsymbol{w}(t)$ is well-defined and entry-wise strictly lower (upper resp.) bounded from $\mathbb{O}_{n}\left(\mathbb{1}_{n}\right.$ resp.), for all $t \in[0,+\infty)$.

Secondly, for any $\boldsymbol{a} \in \Omega$, define a scalar function

$$
V(\boldsymbol{a})=\log \frac{\max _{k} x_{k} / w_{k}(\boldsymbol{a})}{\min _{k} x_{k} / w_{k}(\boldsymbol{a})}
$$

and the following index sets

$$
\begin{aligned}
& \bar{\theta}(\boldsymbol{a})=\left\{i \mid \exists t_{i}>0 \text { s.t. } \frac{x_{i}}{w_{i}(\boldsymbol{a}(t))}=\max _{k} \frac{x_{k}}{w_{k}(\boldsymbol{a}(t))} \text { for any } t \in\left[0, t_{i}\right], \text { with } \boldsymbol{a}(0)=\boldsymbol{a}\right\}, \text { and, } \\
& \underline{\theta}(\boldsymbol{a})=\left\{j \mid \exists t_{j}>0 \text { s.t. } \frac{x_{j}}{w_{j}(\boldsymbol{a}(t))}=\min _{k} \frac{x_{k}}{w_{k}(\boldsymbol{a}(t))} \text { for any } t \in\left[0, t_{j}\right], \text { with } \boldsymbol{a}(0)=\boldsymbol{a}\right\} .
\end{aligned}
$$


Then the right time derivative of $V(\boldsymbol{a}(t))$, along the solution $\boldsymbol{a}(t)$, is given by

$$
\frac{d^{+} V(\boldsymbol{a}(t))}{d t}=a_{j}(t) \phi_{j}(\boldsymbol{a}(t))-a_{i}(t) \phi_{i}(\boldsymbol{a}(t))
$$

for any $i \in \bar{\theta}(\boldsymbol{a}(t))$ and $j \in \underline{\theta}(\boldsymbol{a}(t))$. Define

$$
\begin{aligned}
E & =\left\{\boldsymbol{a} \in \Omega \mid a_{j} \phi_{j}(\boldsymbol{a})-a_{i} \phi_{i}(\boldsymbol{a})=0 \text { for any } i \in \bar{\theta}(\boldsymbol{a}), j \in \underline{\theta}(\boldsymbol{a})\right\}, \\
E_{1} & =\left\{\boldsymbol{a} \in E \mid \boldsymbol{\phi}(\boldsymbol{a})=\mathbb{O}_{n}\right\}, \\
E_{2} & =\left\{\boldsymbol{a} \in E \mid \boldsymbol{\phi}(\boldsymbol{a}) \neq \mathbb{O}_{n}\right\} .
\end{aligned}
$$

One can check that $E$ and $E_{1}$ are compact subsets of $\Omega, E=E_{1} \cup E_{2}$, and $E_{1} \cap E_{2}$ is empty. Denote by $\hat{E}$ the largest invariant subset of $E$. Applying the LaSalle Invariance Principle, see Theorem 3 in [12], we have $\mathcal{D}(\boldsymbol{a}(t), \hat{E}) \rightarrow 0$ as $t \rightarrow+\infty$. Note that, $\lim _{t \rightarrow+\infty} \mathcal{D}(\boldsymbol{a}(t), \hat{E})=0$ does not necessarily leads to $\lim _{t \rightarrow+\infty} \boldsymbol{w}(t)=\boldsymbol{x}$. We need to further refine the result.

For set $E_{1}$, it is straightforward to see that $E_{1} \in \hat{E}$ and $\boldsymbol{w}(\boldsymbol{a})=\boldsymbol{x}$ for any $\boldsymbol{a} \in E_{1}$. Now we prove by contradiction that, if $E_{2} \cap \hat{E}$ is not empty, then, for any $\boldsymbol{a} \in E_{2} \cap \hat{E}$, there exists $i \in \bar{\theta}(\boldsymbol{a})$ such that $a_{i}=0$. Suppose $a_{i}>0$ for any $i \in \bar{\theta}(\boldsymbol{a})$. Since the observation network $G(M)$ is strongly connected, there exists a directed path $i, k_{1}, \ldots, k_{q}, j$ on $G(M)$, where $i \in \bar{\theta}(\boldsymbol{a})$ and $j \in \underline{\theta}(\boldsymbol{a})$. We have $k_{1} \in \bar{\theta}(\boldsymbol{a})$, otherwise, starting with $\tilde{\boldsymbol{a}}(0)=\boldsymbol{a}$, there exists sufficiently small $\Delta t>0$ such that $\phi_{i}(\tilde{\boldsymbol{a}}(t))>0$ and $\tilde{a}_{i}(t)>0$, which contradicts the fact that $\boldsymbol{a}$ is in the largest invariant set of $E$. Repeating this argument, we have $j \in \bar{\theta}(\boldsymbol{a})$, which contradicts $\phi(\boldsymbol{a}) \neq \mathbb{O}_{n}$. Similarly, we have that, for any $\boldsymbol{a} \in E_{2} \cap \hat{E}$, there exists $j \in \underline{\theta}(\boldsymbol{a})$ with $a_{j}=0$.

If the fixed vectors $\boldsymbol{c}$ and $\boldsymbol{x}$ satisfy $\boldsymbol{c}=\boldsymbol{x}$, then there can not exist $\boldsymbol{a} \in E_{2} \cap \hat{E}$ satisfying all the following three properties: i) there exists $i \in \bar{\theta}(\boldsymbol{a})$ such that $a_{i}=0$; ii) there exists $j \in \underline{\theta}(\boldsymbol{a})$ such that $a_{j}=0$; iii) $\boldsymbol{\phi}(\boldsymbol{a}) \neq \mathbb{D}_{n}$. In this case, $E_{2} \cap \hat{E}$ is an empty set, which implies that $\boldsymbol{a}(t) \rightarrow \hat{E}=E_{1}$ and thus $\boldsymbol{w}(t) \rightarrow \boldsymbol{x}$ as $t \rightarrow+\infty$.

Before discussing the case when $\boldsymbol{c} \neq \boldsymbol{x}$, we present some properties of the individual performance measure:

P1: For any $k, l \in\{1, \ldots, n\}, \frac{x_{k}}{c_{k}}\left(1-a_{k}\right) \leq \frac{x_{l}}{c_{l}}\left(1-a_{l}\right)$ leads to $p_{k}(\boldsymbol{a}) \leq p_{l}(\boldsymbol{a})$, and $\frac{x_{k}}{c_{k}}\left(1-a_{k}\right)>\frac{x_{l}}{c_{l}}\left(1-a_{l}\right)$ leads to $p_{k}(\boldsymbol{a})>p_{l}(\boldsymbol{a})$

P2: If there exists $\tau \geq 0$ such that $i \in \bar{\theta}(\boldsymbol{a}(\tau))$ and $a_{i}(\tau)=0$, then $i \in \bar{\theta}(\boldsymbol{a}(t))$ for all $t \geq \tau$;

P3: $\boldsymbol{p}(\boldsymbol{a}(t))$ is finite and strictly bounded from 0 , satisfying

$$
f\left(\frac{x_{i}}{c_{i}}\left(1-\zeta_{i}(\boldsymbol{a}(0))\right)\right) \leq p_{i}(\boldsymbol{a}(t)) \leq f\left(\frac{x_{i}}{c_{i}} \sum_{k} \frac{c_{k}}{\zeta_{k}(\boldsymbol{a}(0))}\right)
$$

with $\zeta_{i}(\boldsymbol{a})$ defined in Theorem 2 (iv).

For the case when $\boldsymbol{c} \neq \boldsymbol{x}$, consider the partition $\varphi_{1}, \ldots, \varphi_{m}$ of the index set $\{1, \ldots, n\}$, with $m \leq n$, satisfying the following two properties:

(i. $x_{k} / c_{k}=x_{l} / c_{l}$ for any $k, l$ in the same subset $\varphi_{r}$;

(ii. $x_{k} / c_{k}>x_{l} / c_{l}$ for any $k \in \varphi_{r}, l \in \varphi_{s}$, with $r<s$.

For any $\boldsymbol{a} \in E_{2} \cap \hat{E}$, since there exists $j \in \underline{\theta}(\boldsymbol{a})$ with $a_{j}=0$, we have $\varphi_{m} \subset \underline{\theta}(\boldsymbol{a})$. For any $i \in \cup_{r=1}^{m-1} \varphi_{r}$, let

$$
\begin{aligned}
E_{2, i}=\left\{\boldsymbol{a} \in \Omega \mid a_{i}=0, a_{j}=0 \text { for any } j \in \varphi_{m},\right. \\
\left.1-\frac{x_{i}}{c_{i}} \frac{c_{k}}{x_{k}} \leq a_{k} \leq 1-\min _{l \in\{1, \ldots, n\}} \frac{x_{l}}{c_{l}} \frac{c_{k}}{x_{k}} \text { for any } k \in \varphi_{1} \cup \cdots \cup \varphi_{m-1} \backslash\{i\}\right\} .
\end{aligned}
$$

With properties P1 and P2 of $\boldsymbol{p}(\boldsymbol{a})$, for any $\boldsymbol{a} \in E_{2, i}$, we have $i \in \bar{\theta}(\boldsymbol{a})$ and $a_{i}=0$. Moreover, 
(i. $E_{2, i} \subset \mathbb{R}^{n}$ is compact for any $i \in \varphi_{1} \cup \cdots \cup \varphi_{m-1}$;

(ii. $\cup_{i \in \varphi_{1}} E_{2, i}, \ldots, \cup_{i \in \varphi_{m-1}} E_{2, i}$ are disjoint and compact subsets of $\mathbb{R}^{n}$;

(iii. $E_{2} \cap \hat{E} \subset \bigcup_{i \in \varphi_{1} \cup \ldots \cup \varphi_{m-1}} E_{2, i}$.

For any $\boldsymbol{a} \in E_{2} \cap \hat{E}$, since there exists $i \in \bar{\theta}(\boldsymbol{a})$ and $j \in \underline{\theta}(\boldsymbol{a})$ such that $a_{i}=a_{j}=0$, on the observation network $G(M)$, there must exists a path $i, k_{1}, \ldots, k_{q}$ satisfying: i) $i \in \bar{\theta}(\boldsymbol{a})$ and $a_{i}=0$; ii) $a_{k_{q}}=0$ and $x_{k_{q}} / c_{k_{q}}<x_{i} / c_{i}$; iii) $a_{k_{l}}>0$ for any $l \in\{1, \ldots, q-1\}$. Consider the trajectory $\tilde{\boldsymbol{a}}(t)$ with $\tilde{\boldsymbol{a}}(0)=\boldsymbol{a}$, we have $\dot{\tilde{a}}_{k_{q-1}} \geq \tilde{a}_{k_{q-1}}\left(1-\tilde{a}_{k_{q-1}}\right)\left(f\left(\frac{x_{k_{q-1}}}{c_{k_{q-1}}}\left(1-\tilde{a}_{k_{q-1}}\right) \sum_{l=1}^{n} \frac{c_{l}}{1-\tilde{a}_{l}}\right) f\left(\left(m_{k_{q-1} k_{q}} \frac{x_{k_{q}}}{c_{k_{q}}}+\left(1-m_{k_{q-1}} k_{q}\right) \frac{x_{i}}{c_{i}}\right) \sum_{l=1}^{n} \frac{c_{l}}{1-\tilde{a}_{l}}\right)\right)$.

The inequality is due to properties P1-P3 of $p_{i}(\boldsymbol{a})$ for $i \in \bar{\theta}(\boldsymbol{a})$ with $a_{i}=0$, and the concavity of the function $f$. Moreover, since $\tilde{a}_{k_{q-1}}$ is strictly bounded from 1 and $\sum_{l} c_{l} /\left(1-\tilde{a}_{l}\right)$ is strictly lower bounded from 0 , there exists $T_{k_{q-1}}(M, \boldsymbol{a}(0), \boldsymbol{a})>0$ such that

$$
p_{k_{q-1}}(\tilde{\boldsymbol{a}}(t))<\frac{2-m_{k_{q-1} k_{q}}}{2} p_{i}(\tilde{\boldsymbol{a}}(t))+\frac{m_{k_{q-1} k_{q}}}{2} p_{k_{q}}(\tilde{\boldsymbol{a}}(t))
$$

Applying the same argument to $k_{q-2}, \ldots, k_{1}$, we have that, there exists $T_{k_{1}}(M, \boldsymbol{a}(0), \boldsymbol{a})>0$ and $\eta_{i k_{1} \ldots k_{q}}(M) \in(0,1)$ such that, for the solution $\tilde{\boldsymbol{a}}(t)$ with $\tilde{\boldsymbol{a}}(0)=\boldsymbol{a}$,

$$
p_{k_{1}}(\tilde{\boldsymbol{a}}(t))<\left(1-\eta_{i k_{1} \ldots k_{q}}(M)\right) p_{i}(\tilde{\boldsymbol{a}}(t))+\eta_{i k_{1} \ldots k_{q}}(M) p_{k_{q}}(\tilde{\boldsymbol{a}}(t))
$$

for all $t \geq T_{k_{1}}(M, \boldsymbol{a}(0), \boldsymbol{a})$. This inequality implies that,

$$
\begin{aligned}
\phi_{i}(\tilde{\boldsymbol{a}}(t)) & \geq m_{i k_{1}} \eta_{i k_{1} \ldots k_{q}}(M)\left(p_{i}(\tilde{\boldsymbol{a}}(t))-p_{k_{q}}(\tilde{\boldsymbol{a}}(t))\right) \\
& \geq m_{i k_{1}} \eta_{i k_{1} \ldots k_{q}}(M) f^{\prime}\left(\frac{x_{i}}{c_{i}}\right) \cdot \sum_{l=1}^{n} \frac{c_{l}}{1-\zeta_{l}(\boldsymbol{a}(0))}\left(\frac{x_{i}}{c_{i}}-\frac{x_{k_{q}}}{c_{k_{q}}}\right)>0 .
\end{aligned}
$$

Since the choices of $i$ and the paths $i, k_{1}, \ldots, k_{q}$ are finite, there exists a constant $\eta>0$ such that, for any $\boldsymbol{a} \in E_{2} \cap \hat{E}$, there exists $T(\boldsymbol{a}(0), \boldsymbol{a})>0$ such that, for any $t \geq T(\boldsymbol{a}(0), \boldsymbol{a})>0$, the solution $\tilde{\boldsymbol{a}}(t)$, with $\tilde{\boldsymbol{a}}(0)=\boldsymbol{a}$, satisfies $i \in \bar{\theta}(\tilde{\boldsymbol{a}}(t))$ and $\phi_{i}(\tilde{\boldsymbol{a}}(t)) \geq \eta>0$.

For any $i \in \varphi_{1} \cup \cdots \cup \varphi_{m-1}$, define

$$
\hat{E}_{2, i}=\left\{\boldsymbol{a} \in E_{2, i} \mid p_{i}(\boldsymbol{a})-\sum_{k=1}^{n} m_{i k} p_{k}(\boldsymbol{a}) \geq \eta\right\}
$$

We have: i) each $\hat{E}_{2, i}$ is a compact subset of $\mathbb{R}^{n}$; ii) $\cup_{i \in \varphi_{1}} \hat{E}_{2, i}, \ldots, \cup_{i \in \varphi_{m-1}} \hat{E}_{2, i}$ are disjoint and compact subsets of $\mathbb{R}^{n}$. Let $\hat{E}_{2}=\cup_{r=1}^{m-1}\left(\cup_{r \in \varphi_{r}} \hat{E}_{2, i}\right)$. For dynamics (6), due to the continuous dependency on the initial condition, for any $\boldsymbol{a} \in\left(E_{2} \cap \hat{E}\right) \backslash\left(\hat{E}_{2} \cap \hat{E}\right)$, there exists $\delta>0$ such that, for any $\tilde{\boldsymbol{a}}(0) \in \mathcal{U}(\boldsymbol{a}, \delta) \cap\left(E_{2} \cap \hat{E}\right)$, where $\mathcal{U}(\boldsymbol{a}, \delta)=\{\boldsymbol{b} \in \Omega \mid \mathcal{D}(\boldsymbol{b}, \boldsymbol{a}) \leq \delta\}, \tilde{\boldsymbol{a}}(t) \in \hat{E}_{2} \cap \hat{E}$ for sufficiently large $t$. Therefore, $\boldsymbol{a}$ can not be an $\omega$-limit point of $\boldsymbol{a}(0)$. We thus obtain that, the $\omega$-limit set of $\boldsymbol{a}(0)$ is in the set $E_{1} \cup\left(\hat{E}_{2} \cap \hat{E}\right)$. Moreover, since $E_{1}, \cup_{i \in \varphi_{1}} \hat{E}_{2, i}, \ldots, \cup_{i \in \varphi_{m-1}} \hat{E}_{2, i}$ are disjoints compact subsets of $\mathbb{R}^{n}$, and the $\omega$-limit set of $\boldsymbol{a}(0)$ is connected and compact, $\boldsymbol{a}(t)$ can only converge to one of the sets $E_{1}, \cup_{i \in \varphi_{1}} \hat{E}_{2, i}, \ldots, \cup_{i \in \varphi_{m-1}} \hat{E}_{2, i}$.

Now we prove $\lim _{t \rightarrow+\infty} \mathcal{D}\left(\boldsymbol{a}(t), E_{1}\right)=0$ by contradiction. Suppose $\omega(\boldsymbol{a}(0)) \in \cup_{i \in \varphi_{r}} \hat{E}_{2, i}$ for some $r \in$ $\{1, \ldots, m-1\}$. Since each $\hat{E}_{2, i}$ is a compact set, there exists $\epsilon>0$ and $\eta(\epsilon)>0$ such that $\phi_{i}(\boldsymbol{a}) \geq \eta(\epsilon)>0$ for any $\boldsymbol{a} \in \mathcal{U}\left(\hat{E}_{2, i}, \epsilon\right)$. For this given $\epsilon>0$, since $\omega(\boldsymbol{a}(0)) \in \cup_{i \in \varphi_{r}} \hat{E}_{2, i}$ leads to $\mathcal{D}\left(\boldsymbol{a}(t), \cup_{i \in \varphi_{r}} \hat{E}_{2, i}\right) \rightarrow 0$ as $t \rightarrow+\infty$, we conclude that, there exists $T>0$ such that, for any $t \geq T, \boldsymbol{a}(t) \in \cup_{i \in \varphi_{r}} \mathcal{U}\left(\hat{E}_{2, i}, \epsilon\right)$. Define 
$V_{r}(\boldsymbol{a})=\min _{i \in \varphi_{r}} a_{i}$, for any $\boldsymbol{a} \in \cup_{i \in \varphi_{r}} \mathcal{U}\left(\hat{E}_{2, i}, \epsilon\right)$. The function $V_{r}(\boldsymbol{a})$ satisfies that, $V_{r}(\boldsymbol{a}) \geq 0$ for any $\boldsymbol{a} \in \cup_{i \in \varphi_{r}} \mathcal{U}\left(\hat{E}_{2, i}, \epsilon\right)$ and $V_{r}(\boldsymbol{a})=0$ if and only if $\boldsymbol{a} \in \cup_{i \in \varphi_{r}} \hat{E}_{2, i}$. Therefore, $\mathcal{D}\left(\boldsymbol{a}(t), \cup_{i \in \varphi_{r}} \hat{E}_{2, i}\right) \rightarrow 0$ leads to $V_{r}(\boldsymbol{a}(t)) \rightarrow 0$ as $t \rightarrow+\infty$. Moreover, since $\boldsymbol{a} \in \mathcal{U}\left(\hat{E}_{2, i}, \epsilon\right)$ for any $i \in \operatorname{argmin}_{k \in \varphi_{r}} a_{k}$, we have

$$
\frac{d^{+} V_{r}(\boldsymbol{a}(t))}{d t}=\min _{i \in \underset{k \in \varphi_{r}}{\operatorname{argmin} a_{k}(t)}} \dot{a}_{i}(t) \geq \delta a_{i}(t)\left(1-a_{i}(t)\right) .
$$

According to Theorem 2(i), for any given $\boldsymbol{a}(0) \in(0,1)^{n}, \boldsymbol{a}(t) \in(0,1)^{n}$ for all $t \geq 0$. Therefore, $d^{+} V_{r}(\boldsymbol{a}(t)) / d t>0$ for all $t \geq T$, which contradicts $\lim _{t \rightarrow+\infty} V_{r}(\boldsymbol{a}(t))=0$. Therefore, we have $\lim _{t \rightarrow+\infty} \mathcal{D}\left(\boldsymbol{a}(t), E_{1}\right)=0$ and $\lim _{t \rightarrow+\infty} \boldsymbol{w}(t)=\boldsymbol{x}$.

Since $\dot{A}(t) \rightarrow \mathbb{O}_{n \times n}$ as $\phi(\boldsymbol{a}(t)) \rightarrow \mathbb{O}_{n}$, there exists an entry-wise non-negative and irreducible matrix $A^{*}$, depending on $A(0)$ and satisfying $\boldsymbol{v}_{\text {left }}\left(A^{*}\right)=\boldsymbol{x}$, such that $A(t) \rightarrow A^{*}$ as $t \rightarrow+\infty$. This concludes the proof.

\section{.5 Proof for Lemma 5}

Since $A(0)$ is primitive and row-stochastic, following the same argument in the proof for Theorem $2(\mathrm{i})$, we have that, there exists $\Delta \tilde{t}>0$ such that, for any $t \in[0, \Delta \tilde{t}]:$ i) $\boldsymbol{w}(t)$ is well-defined and $\boldsymbol{w}(t) \succ \mathbb{O}_{n}$; ii) $A(t)$ is bounded, continuously differentiable to $t$, and satisfies $A(t) \mathbb{1}_{n}=\mathbb{1}_{n}$; iii) $\boldsymbol{p}(\boldsymbol{w}(t))-M \boldsymbol{p}(\boldsymbol{w}(t))$ is bounded. Therefore, for any $t \geq 0$, there exists $\mu$, depending on $t$ and $A(0)$, such that $\dot{A}(t) \succeq \frac{1}{\tau_{\text {ave }}} A^{2}(t)-\left(\frac{1}{\tau_{\text {ave }}}+\mu\right) A(t)$.

Consider the equation $\dot{B}(t)=\frac{1}{\tau_{\text {ave }}} B^{2}(t)-\left(\frac{1}{\tau_{\text {ave }}}+\mu\right) B(t)$, with $B(0)=A(0)$. According to the comparison theorem, $A(t) \succeq B(t)$ for any $t \geq 0$. Let $\boldsymbol{b}_{i}(t)$ be the $i$-th column of $B(t)$ and let $\boldsymbol{y}_{k}(t)=e^{\left(\frac{1}{\tau_{\text {ave }}}+\mu\right) t} \boldsymbol{b}_{k}(t)$. We obtain $\dot{\boldsymbol{y}}_{k}(t)=\frac{1}{\tau_{\text {ave }}} B(t) \boldsymbol{y}_{k}(t)$.

Denote by $\Phi(t, 0)$ the state transition function for the equation $\dot{\boldsymbol{y}}_{k}(t)=\frac{1}{\tau_{\text {ave }}} B(t) \boldsymbol{y}_{k}(t)$, which is written as $\Phi(t, 0)=I_{n}+\sum_{k=1}^{\infty} \Phi_{k}(t)$, where $\Phi_{1}(t)=\int_{0}^{t} B\left(\tau_{1}\right) d \tau_{1}$ and $\Phi_{l}(t)=\int_{0}^{t} B\left(\tau_{1}\right) \int_{0}^{\tau_{1}} \ldots B\left(\tau_{l-1}\right) \int_{0}^{\tau_{l-1}} B\left(\tau_{l}\right) d \tau_{l}$ for $l \geq 2$. By computing the MacLaurin expansion for each $\Phi_{k}(t)$ and summing them together, we obtain that

$$
\Phi(t, 0)=I_{n}+h_{1}(t) B(0)+h_{2}(t) B^{2}(0)+\cdots+h_{n-1}(t) B^{n-1}(0)+O\left(t^{n}\right),
$$

where $h_{k}(t)$ is a polynomial with the form $h_{k}(t)=\eta_{k, k} t^{k}+\eta_{k, k+1} t^{k+1}+\ldots$, and, moreover, $\eta_{k, k}>0$ for any $k \in \mathbb{N}$. Therefore, for $t$ sufficiently small, we have $h_{k}(t)>0$ for any $k \in\{1, \ldots, n-1\}$. Moreover, since $B^{k}(0) \succeq \mathbb{O}_{n \times n}$ for any $k \in \mathbb{N}$ and $B(0)+\cdots+B^{n-1}(0) \succ \mathbb{O}_{n \times n}$, there exists $\Delta t \leq \Delta \tilde{t}$ such that $\Phi(t, 0) \succ \mathbb{O}_{n \times n}$ for any $t \in[0, \Delta t]$.

\section{.6 Discussion on Conjecture 6}

The Monte Carlo method 29] is adopted to estimate the probability that Conjecture 6 holds. For any randomly generated $A(0) \in \operatorname{int}\left(\Delta_{n}\right)$, define the random variable $Z: \operatorname{int}\left(\Delta_{n}\right) \rightarrow\{0,1\}$ as

(i. $Z(A(0))=1$ if there exists $a_{\min }>0$ such that $A(t) \succeq a_{\min } \mathbb{1}_{n} \mathbb{1}_{n}^{\top}$ for all $t \in[0,1000]$;

(ii. $Z(A(0))=0$ otherwise.

Let $p=\mathbb{P}[Z(A(0))=1]$. For $N$ independent random samples $Z_{1}, \ldots, Z_{N}$, in each of which $A(0)$ is randomly generated in $\operatorname{int}\left(\Delta_{n}\right)$, define $\hat{p}_{N}=\sum_{i=1}^{N} Z_{i} / N$. For any accuracy $\epsilon \in(0,1)$ and confidence level $1-\xi \in(0,1)$, $\left|\hat{p}_{N}-p\right|<\epsilon$ with probability greater than $1-\xi$ if

$$
N \geq \frac{1}{2 \epsilon^{2}} \log \frac{2}{\xi}
$$


For $\epsilon=\xi=0.01$, the Chernoff bound (11) is satisfied by $N=27000$. We run 27000 independent MATLAB simulations of the assign.appraise/influence dynamics with $n=7$ and find that $\hat{p}_{N}=1$. Therefore, for any $A(0) \in \operatorname{int}\left(\Delta_{n}\right)$, with $99 \%$ confidence level, there is at least 0.99 probability that $A(t)$ is entry-wise strictly lower bounded from $\mathbb{0}_{n \times n}$ for all $t \in[0,10000]$.

Moreover, we present in the following lemma a sufficient condition for Conjecture 6 on the initial appraisal matrix $A(0)$ and the parameters $\tau_{\text {ave }}, \tau_{\text {app }}$.

Lemma 9 (Strictly positive lower bound of appraisals). Consider the assign/appraise/influence dynamics (9), based on Assumptions 3, 6, with the assignment $\boldsymbol{w}(t)$ and performance $\boldsymbol{p}(\boldsymbol{w})$ as in Assumptions 1 and 2 respectively. For any initial appraisal matrix $A(0)$ that is entry-wise positive and row-stochastic, as long as

$$
\frac{\tau_{\text {app }}}{\tau_{\text {ave }}} \geq \frac{1-\xi_{0}}{\xi_{0}}\left(f\left(\frac{x_{\max }}{\xi_{0}}\right)-f\left(\frac{x_{\min }}{1-(n-1) \xi_{0}}\right)\right),
$$

where the constant $\xi_{0}$ is defined as in Theorem 0 (ii), then there exists $a_{\min }>0$ such that $A(t) \succeq a_{\min } \mathbb{1}_{n} \mathbb{1}_{n}^{\top}$.

Proof. First of all, by definition we have $w_{s}(t)=\sum_{k} w_{k}(t) a_{k s}(t)$. The right-hand side of this equation is a convex combination of $\left\{a_{1 s}(t), \ldots, a_{n s}(t)\right\}$. Therefore, $\max _{k} a_{k s}(t) \geq w_{s}(t) \geq \xi_{0}$ for all $t \in[0,+\infty)$.

At any time $t \geq 0$, for any pair $(i, j)$ such that $a_{i j}(t)=\min _{k, l} a_{k l}(t)$, the dynamics for $a_{i j}(t)$ is

$$
\dot{a}_{i j}(t)=\frac{1}{\tau_{\text {ave }}}\left(\sum_{k} a_{i k}(t) a_{k j}(t)-a_{i j}(t)\right)-\frac{1}{\tau_{\text {app }}} a_{i i}(t) a_{i j}(t)\left(p_{i}(\boldsymbol{w}(t))-\sum_{k=1}^{n} m_{i k} p_{k}(\boldsymbol{w}(t))\right) .
$$

For simplicity, in this proof, denote $\phi_{i}=p_{i}(\boldsymbol{w}(t))-\sum_{k=1}^{n} m_{i k} p_{k}(\boldsymbol{w}(t))$. Suppose $a_{m j}(t)=\max _{k} a_{k j}(t)$. We have

$$
\dot{a}_{i j}(t) \geq \frac{1}{\tau_{\text {ave }}} a_{i j}(t) a_{m j}(t)-\frac{1}{\tau_{\text {ave }}} a_{i j}^{2}(t)-\frac{1}{\tau_{\text {app }}} a_{i i}(t) a_{i j}(t) \phi_{i}
$$

Therefore,

$$
\frac{\dot{a}_{i j}}{a_{i j}} \geq \frac{1}{\tau_{\mathrm{ave}}} \xi_{0}-\frac{1}{\tau_{\mathrm{app}}}\left(1-\xi_{0}\right)\left(f\left(\frac{x_{\max }}{\xi_{0}}\right)-f\left(\frac{x_{\min }}{1-(n-1) \xi_{0}}\right)\right) .
$$

The condition on $\frac{1}{\tau_{\text {ave }}} / \frac{1}{\tau_{\text {app }}}$ in Lemma 9 guarantees that $\dot{a}_{i j}(t) / a_{i j}(t)$ is positive if $a_{i j}(t)=\min _{k, l} a_{k l}(t)$. This concludes the proof.

\section{.7 Proof for Theorem 7}

Statement (i) is proved following the same argument in the proof for Theorem 2 (i). For any given $A(0)$ that is row-stochastic and entry-wise positive, the closed and bounded invariant set $\Omega$ for $A(t)$ is given by $\Omega=\left\{A \in \mathbb{R}^{n \times n} \mid A \succ a_{\min } \mathbb{1}_{n} \mathbb{1}_{n}^{\top}, A \mathbb{1}_{n}=\mathbb{1}_{n}\right\}$, where $a_{\min }>0$ is given by Conjecture 6 .

Since $\boldsymbol{w}^{\top}(t)\left(A^{2}(t)-A(t)\right)=\mathbb{O}_{n}^{\top}$ for all $t \geq 0$, we conclude that, $\boldsymbol{w}(t)$ in the assign/appraise/influence dynamics also obeys the generalized replicator dynamics (7). Consider $\boldsymbol{w}(t)$ as a function of $A(t)$. Define $\phi(A)=\boldsymbol{p}(\boldsymbol{w}(A))-M \boldsymbol{p}(\boldsymbol{w}(A))$ and

$$
V(A)=\log \frac{\max _{k} x_{k} / w_{k}(A)}{\min _{k} x_{k} / w_{k}(A)}
$$

For any $t \in[0,+\infty)$, there exists $i \in \operatorname{argmax}_{k} x_{k} / w_{k}(A(t))$ and $j \in \operatorname{argmin}_{k} x_{k} / w_{k}(A(t))$ such that $V(A(t))=\log \left(x_{i} w_{j}(A(t)) / x_{j} w_{i}(A(t))\right)$, and $\frac{d^{+} V(A)}{d t}=a_{j j} \phi_{j}(A)-a_{i i} \phi_{i}(A) \leq 0$. Therefore, $V(A(t))$ is non-increasing with $t$, which in turn implies

$$
\frac{x_{i}}{x_{j}} \frac{w_{j}(t)}{w_{i}(t)} \leq \frac{\max _{k} x_{k} / w_{k}(0)}{\min _{k} x_{k} / w_{k}(0)}=\gamma_{0}
$$


for any $i, j \in\{1, \ldots, n\}$. This inequality, combined with the fact that $\sum_{k} w_{k}(t)=1$ for any $t \geq 0$, leads to the inequalities in statement (ii).

Similar to the proof for Theorem 3 , define

$$
\begin{aligned}
& \bar{\theta}(A)=\left\{i \mid \exists t_{i}>0 \text { s.t. } \frac{x_{i}}{w_{i}(A(t))}=\max _{k} \frac{x_{k}}{w_{k}(A(t))} \text { for any } t \in\left[0, t_{i}\right] \text { with } A(0)=A\right\}, \\
& \underline{\theta}(A)=\left\{j \mid \exists t_{j}>0 \text { s.t. } \frac{x_{j}}{w_{j}(A(t))}=\min _{k} \frac{x_{k}}{w_{k}(A(t))} \text { for any } t \in\left[0, t_{j}\right] \text { with } A(0)=A\right\},
\end{aligned}
$$

and let $E=\left\{A \in \Omega \mid d^{+} V(A) / d t=0\right\}$. For any $A \in E$, since $A \succeq a_{\min } \mathbb{1}_{n} \mathbb{1}_{n}^{\top}$, we have $\phi_{i}(A)=\phi_{j}(A)=0$ for any $i \in \bar{\theta}(A)$ and $j \in \underline{\theta}(A)$. Suppose individual $s$ is a globally reachable node in the observation network. There exists a directed path $i, k_{1}, \ldots, k_{q}, s$. Without loss of generality, suppose $q \geq 1$. For any $A$ in the largest invariant subset of $E$, we have $k_{1} \in \bar{\theta}(A)$ and therefore $\phi_{k_{1}}(A)=0$. This iteration of argument leads to $s \in \bar{\theta}(A)$. Following the same line of argument, we have $s \in \underline{\theta}(A)$. Therefore, for any given $A(0) \succ \mathbb{O}_{n \times n}$ that is row-stochastic, the solution $A(t)$ converges to $\hat{E}=\left\{A \in \Omega \mid \phi(A)=\mathbb{O}_{n}\right\}=\left\{A \in \Omega \mid \boldsymbol{v}_{\text {left }}(A)=\boldsymbol{x}\right\}$.

Let $\tilde{A}=\max _{j}\left(\max _{k} a_{k j}-\min _{k} a_{k j}\right)$. One can check that $d^{+} \tilde{V}(A) / d t$ along the dynamics (9) is a continuous function of $A$ for any $A \in \Omega$. Define $\hat{E}_{\epsilon / 2}=\left\{A \in \hat{E} \mid\left\|A-\mathbb{1}_{n} \boldsymbol{x}^{\top}\right\|_{2} \geq \epsilon / 2\right\}$. Since $\hat{E}$ is compact, $\hat{E}_{\epsilon / 2}$ is also a compact set. For any $A \in \hat{E}_{\epsilon / 2}$, since $d^{+} \tilde{V}(A) / d t$ is strictly negative and depends continuously on $A$, there exists a neighborhood $\mathcal{U}\left(A, r_{A}\right)=\left\{\tilde{A} \in \Omega \mid\|\tilde{A}-A\|_{2} \leq r_{A}\right\}$ such that $d^{+} \tilde{V}(\tilde{A}) / d t<0$ for any $\tilde{A} \in \mathcal{U}\left(A, r_{A}\right)$. Due to the compactness of $\hat{E}_{\epsilon / 2}$ and according to the Heine-Borel finite cover theorem, there exists $K \in \mathbb{N}$ and $\left\{A_{k}, r_{k}\right\}_{k \in\{1, \ldots, K\}}$, where $A_{k} \in \hat{E}_{\epsilon / 2}$ and $r_{k}>0$ for any $k \in\{1, \ldots, K\}$, such that $\hat{E}_{\epsilon / 2} \subset \cup_{k=1}^{K} \mathcal{U}\left(A_{k}, r_{k}\right)$.

Define the distance $\mathcal{D}: \mathbb{R}^{n} \times \mathbb{R}^{n} \rightarrow \mathbb{R}_{\geq 0}$ as in the proof for Theorem 3 . Let $\delta=\min \left\{r_{1}, \ldots, r_{k}, \epsilon / 2\right\}$ and

$$
\begin{aligned}
& B_{1}=\left\{A \in \Omega \mid \mathcal{D}(A, \hat{E}) \leq \delta, \mathcal{D}\left(A, \hat{E}_{\epsilon / 2}\right)>\delta\right\}, \\
& B_{2}=\left\{A \in \Omega \mid \mathcal{D}(A, \hat{E}) \leq \delta, \mathcal{D}\left(A, \hat{E}_{\epsilon / 2}\right) \leq \delta\right\} .
\end{aligned}
$$

We have $B_{1} \cap B_{2}$ is empty. For any $A \in B_{1}$, since $\mathcal{D}(A, \hat{E}) \leq \delta, \mathcal{D}\left(A, \hat{E}_{\epsilon / 2}\right)>\delta$, there exists $\tilde{A} \in \hat{E}_{\epsilon / 2}$ such that $\mathcal{D}(A, \tilde{A}) \leq \delta$. Since $\mathcal{D}\left(\tilde{A}, \mathbb{1}_{n} \boldsymbol{x}^{\top}\right)<\epsilon / 2$, we have $\mathcal{D}\left(A, \mathbb{1}_{n} \boldsymbol{x}^{\top}\right) \leq \mathcal{D}(A, \tilde{A})+\mathcal{D}\left(\tilde{A}, \mathbb{1}_{n} \boldsymbol{x}^{\top}\right)<\epsilon$. Therefore, $B_{1} \subset \mathcal{U}\left(\mathbb{1}_{n} \boldsymbol{x}^{\top}, \epsilon\right)$. Moreover, since $B_{2}$ is compact, $\tilde{V}(A)$ is lower bounded and $d^{+} \tilde{V}(A) / d t$ is strictly upper bounded from 0 in $B_{2}$. Since $\lim _{t \rightarrow+\infty} \mathcal{D}(A(t), \hat{E})=0$, there exists $t_{0}>0$ such that $A(t) \in B_{1} \cup B_{2}$ for any $t \geq 0$. Therefore, for any $t \geq t_{0}$, there exists $t_{1} \geq t$ such that $A\left(t_{1}\right) \in B_{1}$. This argument is valid for any $\epsilon>0$, which implies that $\mathbb{1}_{n} \boldsymbol{x}^{\top}$ is an $\omega$-limit point for any given $A(0)$.

Since $\hat{E}$ is compact, $\mathcal{D}(A, \tilde{E})$ is strictly positive. Since $\lim _{t \rightarrow+\infty} \mathcal{D}(A(t), \hat{E})=0$, any $A \in \Omega \backslash \hat{E}$ can not be an $\omega$-limit point of $A(0)$. For any $A \in \hat{E} \backslash\left\{\mathbb{1}_{n} \boldsymbol{x}^{\top}\right\}$, since the solution passing through $A$ asymptotically converges to $\mathbb{1}_{n} \boldsymbol{x}^{\top}, A \in \hat{E} \backslash\left\{\mathbb{1}_{n} \boldsymbol{x}^{\top}\right\}$ can not be an $\omega$-limit point of $A(0)$ either. Therefore, the $\omega$-limit set of $A(0)$ is $\left\{\mathbb{1}_{n} \boldsymbol{x}^{\top}\right\}$. This concludes the proof.

\section{References}

[1] E. G. Anderson Jr and K. Lewis. A dynamic model of individual and collective learning amid disruption. Organization Science, 25(2):356-376, 2013.

[2] X. Chen, J. Liu, Z. Xu, and T. Başar. Distributed evaluation and convergence of self-appraisals in social networks. In IEEE Conf. on Decision and Control, pages 2895-2900, Osaka, Japan, 2015.

[3] R. Cressmana and Y. Tao. The replicator equation and other game dynamics. Proceedings of the National Academy of Sciences, 111:10810-10817, 2014. 
[4] M. H. DeGroot. Reaching a consensus. Journal of the American Statistical Association, 69(345):118-121, 1974.

[5] L. Festinger. A theory of social comparison processes. Human Relations, 7(2):117-140, 1954.

[6] M. J. Fox, B. Touri, and J. S. Shamma. Dynamics in atomic signaling games. Journal of Theoretical Biology, 376:82-90, 2015.

[7] N. E. Friedkin. A formal theory of reflected appraisals in the evolution of power. Administrative Science Quarterly, 56(4):501-529, 2011.

[8] N. E. Friedkin and C. E. Johnsen. Attitude change, affect control, and expectation states in the formation of influence networks. Advances in Group Processes, 20:1-29, 2003.

[9] A. Jadbabaie, A. Sandroni, and A. Tahbaz-Salehi. Non-Bayesian social learning. Games and Economic Behavior, 76(1):210-225, 2012.

[10] P. Jia, N. E. Friedkin, and F. Bullo. The coevolution of appraisal and influence networks leads to structural balance. IEEE Transactions on Network Science and Engineering, July 2016. To appear.

[11] P. Jia, A. MirTabatabaei, N. E. Friedkin, and F. Bullo. Opinion dynamics and the evolution of social power in influence networks. SIAM Review, 57(3):367-397, 2015.

[12] J. P. LaSalle. Stability theory for ordinary differential equations. Journal of Differential Equations, 4:57-65, 1968.

[13] D. Lazer and A. Friedman. The network structure of exploration and exploitation. Administrative Science Quarterly, 52(4):667-694, 2007.

[14] J. Y. Lee, D. G. Bachrach, and K. Lewis. Social network ties, transactive memory, and performance in groups. Organization Science, 25(3):951-967, 2014.

[15] K. Lewis. Measuring transactive memory systems in the field: scale development and validation. Journal of Applied Psychology, 88(4):587, 2003.

[16] K. Lewis. Knowledge and performance in knowledge-worker teams: A longitudinal study of transactive memory systems. Management Science, 50:1519-1533, 2004.

[17] D. W. Liang, R. Moreland, and L. Argote. Group versus individual training and group performance: The mediating role of transactive memory. Personality and Social Psychology Bulletin, 21:384-393, 1995.

[18] J. Lorenz and D. A. Lorenz. On conditions for convergence to consensus. IEEE Transactions on Automatic Control, 55:1651-1656, 2010.

[19] D. Madeo and C. Mocenni. Game interactions and dynamics on networked populations. IEEE Transactions on Automatic Control, 60:1801-1810, 2015.

[20] J. G. March. Exploration and exploitation in organizational learning. Organization Science, 2(1):71-87, 1991.

[21] J. R. Marden, H. P. Young, G. Arslan, and J. S. Shamma. Payoff-based dynamics for multiplayer weakly acyclic games. SIAM Journal on Control and Optimization, 48(1):373-396, 2009. 
[22] S. A. Marvel, J. Kleinberg, R. D. Kleinberg, and S. H. Strogatz. Continuous-time model of structural balance. Proceedings of the National Academy of Sciences, 108(5):1771-1776, 2011.

[23] W. Mason and D. J. Watts. Collaborative learning in networks. Proceedings of the National Academy of Sciences, 109(3):764-769, 2011.

[24] W. Mei, N. E. Friedkin, K. Lewis, and F. Bullo. Dynamic models of appraisal networks explaining collective learning. In IEEE Conf. on Decision and Control, Las Vegas, NV, USA, December 2016. To appear.

[25] A. MirTabatabaei and F. Bullo. Opinion dynamics in heterogeneous networks: Convergence conjectures and theorems. SIAM Journal on Control and Optimization, 50(5):2763-2785, 2012.

[26] J. Morand-Ferron and J. L. Quinn. Larger groups of passerines are more efficient problem solvers in the wild. Proceedings of the National Academy of Sciences, 108(38):15898-15903, 2011.

[27] E. T. Palazzolo. Organizing for information retrieval in transactive memory systems. Communication Research, 32(6):726-761, 2005.

[28] W. H. Sandholm. Population Games and Evolutionary Dynamics. MIT Press, 2010.

[29] R. Tempo, G. Calafiore, and F. Dabbene. Randomized Algorithms for Analysis and Control of Uncertain Systems. Springer, 2005.

[30] V. A. Traag, P. Van Dooren, and P. De Leenheer. Dynamical models explaining social balance and evolution of cooperation. PLOS ONE, 8(4):e60063, 2013.

[31] D. M. Wegner, T. Giuliano, and P. T. Hertel. Cognitive interdependence in close relationships. In J. Ickes, editor, Compatible and Incompatible Relationships, pages 253-276. Springer, 1985.

[32] J. H. Wilkinson. The Algebraic Eigenvalue Problem. Oxford University Press, 1965.

[33] A. W. Woolley, C. F. Chabris, A. Pentland, N. Hasnmi, and T. W. Malone. Evidence for a collective intelligence factor in the performance of human groups. Science, 330:686-688, 2010.

[34] S. Wuchty, B. F. Jones, and B. Uzzi. The increasing dominance of teams in production of knowledge. Science, 316:1036-1039, 2007.

[35] Y. C. Yuan, I. Carboni, and K. Ehrlich. The impact of awareness and accessibility on expertise retrieval: A multilevel network perspective. Journal of the American Society for Information Science and Technology, 61(4):700-714, 2010. 